\title{
Difference and symmetric difference for intuitionistic fuzzy sets
}

\author{
Taiwo Enayon Sunday ${ }^{1}$, Romuald Dzati Kamga ${ }^{2}$, \\ Siméon Fotso ${ }^{3}$ and Louis Aimé Fono ${ }^{4}$ \\ ${ }^{1}$ Systems Engineering and Engineering Management, City University of Hong Kong \\ Tat Chee Avenue, Hong Kong \\ e-mail: estaiwo2-c@my.cityu.edu.hk \\ ${ }^{2}$ Laboratoire de Mathématiques Appliquées-UFRD MIBA, Université de Yaoundé I \\ B.P. 812 Yaoundé, Cameroun \\ e-mail: romualdkamgal@yahoo.fr \\ ${ }^{3}$ ENS Yaoundé - Université de Yaoundé I \\ B.P. 47 Yaoundé, Cameroun \\ e-mail: simeonfotso@yahoo.fr \\ ${ }^{4}$ Laboratoire de Mathématiques, Université de Douala \\ B.P. 24157 Douala, Cameroun \\ e-mail: Ifono2000@yahoo.fr
}

Received: 31 March 2018 Revised: 24 November $2018 \quad$ Accepted: 1 December 2018

\begin{abstract}
Fono et al. [10] determined some classes of difference and symmetric difference operations for fuzzy sets using fuzzy implication operators. Intuitionistic fuzzy sets are known to be generalizations of fuzzy sets. So, in this paper, we propose new difference and symmetric difference operations for intuitionistic fuzzy sets based on intuitionistic fuzzy R-implication operators and standard intuitionistic fuzzy negation operator. We establish that some common properties of the difference operations for fuzzy sets established earlier by Fono et al. in [10] and for crisp sets are preserved by the new obtained operations for intuitionistic fuzzy sets. We display a specific property satisfied by difference operation in crisp and fuzzy cases and violated in intuitionistic fuzzy case. The proposed difference and symmetric difference operations for intuitionistic fuzzy sets generalize the case for fuzzy sets. This strength provides a more dynamic perspective into the studies and applications of these operations.
\end{abstract}


Keywords: Intuitionistic fuzzy set, Difference operation, Symmetric difference operation, Intuitionistic fuzzy R-implication, Intuitionistic fuzzy negation.

2010 Mathematics Subject Classification: 03F55.

\section{Introduction}

The framework of fuzzy set provides us with tools to handle problems in which the source of vagueness is the ambiguity in criteria of class membership rather than randomness [20]. In this framework, any element of a universal crisp set is allowed to belong to a subset partially with a membership grade usually between 0 and 1 assigned to it. Furthermore, the sum of the membership grade and non-membership grade of an element is always 1 . But in reality, this case is not always true because there may be some hesitation degree [9] and this led to the introduction of intuitionistic fuzzy sets as generalization of fuzzy sets by Atanassov [1] in which the degree of hesitation is accounted for, so that the sum of the membership grade, non-membership grade of an element and its degree of hesitation is always 1 . Throughout this paper, we consider that definition of an intuitionistic fuzzy set.

Since intuitionistic fuzzy set theory is a generalization of the fuzzy set theory, a rigorous study was needful to be able to establish workable results when concepts under crisp sets and fuzzy sets are transferred. A new set of definitions for set operations needed to be proposed for this field. Many standard operations (such as inclusion, intersection, union, complement, etc) $[2,7,9,11,12]$ have been unanimously agreed upon to serve as usual operations on intuitionistic fuzzy sets. Meanwhile, the need to study these operations in a more mathematical framework which allows for generalization has motivated many scholars [3-6, 11-13,17-19] among others to undertake studies in intuitionistic fuzzy operators and generators. Of these operators which are germane to establishing results in our current research include intuitionistic fuzzy t-norms, t-conorms, R-implications, co-implications and negations. Cornelis et al. [5, 6] and Atanassov [3] have established many results in the study of intuitionistic fuzzy implications, co-implications, negations and their properties. Some of these results have provided in great measure some required mathematical background for our current study.

Fono et al. [10] have proposed two classes of difference operations for fuzzy sets and two classes of symmetric difference for fuzzy sets using the fuzzy implication operators. They established that these difference and symmetric difference operations for fuzzy sets of type 1 and 2 preserve the classical properties of difference and symmetric difference operations for crisp sets. Inspired by their work on fuzzy sets, we introduce new definitions for difference and symmetric difference for intuitionistic fuzzy sets by means of intuitionistic fuzzy R-implications and we study their properties.

Huawen [15] defined three difference operations for intuitionistic fuzzy sets, one based on the intuitionistic fuzzy t-norm $\mathcal{T}_{M}=(\min , \max )$ and the remaining based on any decreasing intuitionistic fuzzy generators as follows: For any two intuitionistic fuzzy sets $A$ and $B$ of $X$, 


$$
\begin{aligned}
& A-{ }_{1} B=\left\{\left\langle x, \mu_{A}(x) \wedge \nu_{B}(x), \nu_{A}(x) \vee \mu_{B}(x)\right\rangle \mid x \in X\right\}, \\
& A-{ }_{2} B=\left\{\left\langle x, \mu_{A}(x) \wedge \varphi\left(1-\nu_{B}(x)\right), \nu_{A}(x) \vee\left(1-\varphi\left(\mu_{B}(x)\right)\right)\right\rangle \mid x \in X\right\}, \\
& A-{ }_{3} B=\left\{\left\langle x, \mu_{A}(x) \wedge \varphi\left(1-\nu_{B}(x)\right), \nu_{A}(x) \vee \varphi\left(1-\mu_{B}(x)\right)\right\rangle \mid x \in X\right\},
\end{aligned}
$$

where $\varphi$ is any decreasing intuitionistic fuzzy generator such that $\varphi(0)=1$.

However, these definitions do not provide a sufficient endowment to explore the mathematical extensions of these operations to the more general cases that apply to any intuitionistic fuzzy t-norm. Huawen's difference operations preserve only two out of the four properties which are the minimal conditions (as we have established in the results of Proposition 2) to require for a difference operation on sets, fuzzy sets and intuitionistic fuzzy sets in general. It is worthy to note here that the Huawen's difference operation $-_{1}$ is trivially the generalization of the difference operation in the sense of crisp set given by $A-B=A \cap B^{c}$. As he noted, if we choose the generator $\varphi$ to be the standard negator defined by $\varphi(x)=1-x$, then the difference operations $-{ }_{2}$ and -3 reduce to $-_{1}$. The complement functions (which are special examples of any difference operation) constructed from the difference operations $-_{2}$ and $-_{3}$ in Equations (2) and (3) are the same as the intuitionistic fuzzy complementation and intuitionistic fuzzy pseudo-complementation respectively, obtained by Bustince, et al. [4]. Thus, we can refer to the difference operation $-{ }_{3}$ as intuitionistic fuzzy pseudo-difference operation, which in general does not inherit the general properties of the difference $-{ }_{2}$.

It is also notable to remark here that, the intuitionistic fuzzy complementation associated to difference $-{ }_{2}$ defined by Huawen [15] and Bustince, et al. [4] depends largely on the choice of the intuitionistic fuzzy generator. Thus, with different choice of intuitionistic fuzzy generator, the intuitionistic fuzzy complementation so defined may yield different result. Meanwhile, the intuitionistic fuzzy complementation associated to the difference operation we proposed, though by means of intuitionistic fuzzy R-implications, yet yields the same result for any choice of associated t-representable intuitionistic fuzzy t-norm.

By these new difference and symmetric difference operations for intuitionistic fuzzy sets we have proposed, we are able to construct typical examples of intuitionistic fuzzy difference and symmetric difference associated to the three usual known of intuitionistic fuzzy t-norms (the minimum, product and Lukasiewicz). More explicit examples of these new operations can be constructed for other t-representable intuitionistic fuzzy t-norms. This possibility provides a more robust knowledge and insight into the study of these operations in general cases and their applications would be more enriched.

The rest of this paper is organized as follows. Section 2 recalls some preliminaries on fuzzy sets and intuitionistic fuzzy sets. It also recalls known and useful results on difference and symmetric difference of fuzzy sets established by Fono et al. [10]. Section 3 introduces difference and symmetric difference of intuitionistic fuzzy sets and establishes their properties. Section 4 gives some concluding remarks. An Appendix recalls some known results of the fuzzy case that we use. 


\section{Preliminaries}

Throughout this paper, $X$ shall denote a nonempty universal set, $\top$ a t-norm and $S$ a t-conorm.

In this Section, we introduce some basic definitions and provide some preliminary results needed in the rest of the paper. Some other useful notions and concepts on fuzzy sets are recalled in Appendix.

\subsection{Intuitionistic fuzzy sets, intuitionistic fuzzy operators and operations}

Here, we introduce the basic concepts of intuitionistic fuzzy sets, recall the definitions and examples of some intuitionistic fuzzy operators and operations $([5,13,17])$.

Definition 1 (Intuitionistic Fuzzy Set $[7,9,17])$. An intuitionistic fuzzy set $D$ on $X$ is defined by:

$$
D=\left\{\left(x, \mu_{D}(x), \nu_{D}(x)\right) \mid \mu_{D}(x), \nu_{D}(x) \in[0,1], 0 \leq \mu_{D}(x)+\nu_{D}(x) \leq 1, \forall x \in X\right\},
$$

where $\mu_{D}(x), \nu_{D}(x)$ are the degrees of membership and non-membership of $x$ in $D$, respectively.

If $\mu_{D}(x)+\nu_{D}(x)=1$, then $D$ is a fuzzy set of $X$ where $\mu_{D}(x)$ is the degree of membership of $x$ in $D$.

We will subsequently be referring to the complete lattice $\left(L^{*}, \leq_{L^{*}}\right)$ with $0_{L^{*}}=(0,1)$ and $1_{L^{*}}=(1,0)$ as the units where $L^{*}=\left\{\left(x_{1}, x_{2}\right) \mid\left(x_{1}, x_{2}\right) \in[0,1] \times[0,1], x_{1}+x_{2} \leq 1\right\}$ and $\leq_{L^{*}}$ is an order on $L^{*}$ defined by: for all $\left(x_{1}, x_{2}\right),\left(y_{1}, y_{2}\right) \in L^{*},\left(x_{1}, x_{2}\right) \leq_{L^{*}}\left(y_{1}, y_{2}\right)$ if and only if $x_{1} \leq$ $y_{1}$ and $x_{2} \geq y_{2}$. The meet operator $\wedge$ and the join operator $\vee$ on this lattice, $\left(L^{*}, \leq_{L^{*}}\right)$ are defined for all $\left(x_{1}, x_{2}\right),\left(y_{1}, y_{2}\right) \in L^{*}$ as:

$$
\begin{aligned}
& \left(x_{1}, x_{2}\right) \wedge\left(y_{1}, y_{2}\right)=\left(\min \left(x_{1}, y_{1}\right), \max \left(x_{2}, y_{2}\right)\right) \\
& \left(x_{1}, x_{2}\right) \vee\left(y_{1}, y_{2}\right)=\left(\max \left(x_{1}, y_{1}\right), \min \left(x_{2}, y_{2}\right)\right) .
\end{aligned}
$$

Definition 2 (Intuitionistic Fuzzy t-norm and t-conorm, (see [5,6,13])). 1. An intuitionistic fuzzy t-norm is a binary operation $\mathcal{T}: L^{*} \times L^{*} \longrightarrow L^{*}$ such that for any $\mathrm{x} \in L^{*}, \mathcal{T}\left(\mathrm{x}, 1_{L^{*}}\right)=\mathbf{x}$ (neutral element) and, $\mathcal{T}$ satisfies commutativity, monotonicity (increasing) and associativity.

2. An intuitionistic fuzzy t-conorm is a binary operation $\mathcal{J}: L^{*} \times L^{*} \longrightarrow L^{*}$ such that for any $\mathrm{x} \in L^{*}, \mathcal{J}\left(\mathrm{x}, 0_{L^{*}}\right)=\mathrm{x}$ and, $\mathcal{J}$ is commutative, monotone increasing and associative.

Definition 3 (Intuitionistic Fuzzy Negation, (see [5-7, 18])). An intuitionistic fuzzy negation is a non-increasing mapping $\mathcal{N}: L^{*} \longrightarrow L^{*}$ satisfying $\mathcal{N}\left(0_{L^{*}}\right)=1_{L^{*}}$ and $\mathcal{N}\left(1_{L^{*}}\right)=0_{L^{*}}$. If $\mathcal{N}(\mathcal{N}(\mathbf{x}))=\mathbf{x}, \forall \mathbf{x} \in L^{*}$, then $\mathcal{N}$ is said to be involutive. An involutive intuitionistic fuzzy negation is called strong intuitionistic fuzzy negation.

Deschrijver et al. and, Reseir and Bedregal $[6,18]$ have shown that an involutive intuitionistic fuzzy negation, $\mathcal{N}$, can be characterized by an involutive fuzzy negation by proving that, 
if the fuzzy negation $N$ is involutive, then $\mathcal{N}(\mathbf{x})=\left(N\left(1-x_{2}\right), 1-N\left(x_{1}\right)\right)$. An example of a strong (involutive) intuitionistic fuzzy negation is the standard negation $\mathcal{N}_{s}$ on $L^{*}$ defined by $\mathcal{N}_{s}\left(x_{1}, x_{2}\right)=\left(x_{2}, x_{1}\right)$.

We now recall useful classes of intuitionistic fuzzy t-norm and t-conorm and, their implications and co-implications.

Definition 4 (t-Representable intuitionistic fuzzy t-norm and t-conorm (see [5, 6, 13, 17])). An intuitionistic fuzzy t-norm $\mathcal{T}$ (respectively intuitionistic fuzzy t-conorm $\mathcal{J}$ ) is $t$-representable if there exists a fuzzy $t$-norm $T$ and a fuzzy $t$-conorm $S$ (respectively a fuzzy $t$-conorm $S^{\prime}$ and a fuzzy t-norm $\left.\top^{\prime}\right)$ such that, for all $\mathbf{x}=\left(x_{1}, x_{2}\right), \mathbf{y}=\left(y_{1}, y_{2}\right) \in L^{*}, \mathcal{T}(\mathbf{x}, \mathbf{y})=\left(\top\left(x_{1}, y_{1}\right), S\left(x_{2}, y_{2}\right)\right)$ and $\mathcal{J}(\mathbf{x}, \mathbf{y})=\left(S^{\prime}\left(x_{1}, y_{1}\right), \top^{\prime}\left(x_{2}, y_{2}\right)\right)$, respectively.

The following result allows us to construct t-representable intuitionistic fuzzy t-norms and $\mathrm{t}$-conorms from fuzzy t-norms and t-conorms.

Theorem 1. [5, 6, 17] Given a fuzzy $t$-norm $\top$ and fuzzy $t$-conorm $S$ satisfying $\top(a, b) \leq 1-$ $S(1-a, 1-b)$ for all $a, b \in[0,1]$, then $\mathcal{T}(\mathbf{x}, \mathbf{y})=\left(\top\left(x_{1}, y_{1}\right), S\left(x_{2}, y_{2}\right)\right)$ and $\mathcal{J}(\mathbf{x}, \mathbf{y})=$ $\left(S\left(x_{1}, y_{1}\right), \top\left(x_{2}, y_{2}\right)\right)$ for all $\mathbf{x}=\left(x_{1}, x_{2}\right), \mathbf{y}=\left(y_{1}, y_{2}\right) \in L^{*}$, are t-representable intuitionistic fuzzy t-norm and t-representable intuitionistic fuzzy $t$-conorm respectively.

We denote by IF-t-norm the intuitionistic fuzzy t-norm and, by IF-t-conorm the intuitionistic fuzzy t-conorm.

Definition 5 (Intuitionistic fuzzy R-implication and co-implicator [5,6,13]). 1. An intuitionistic fuzzy R-implication (for short, IF-R-implication) associated with an IF-t-norm, $\mathcal{T}=$ $(\top, S)$, is a mapping $I_{\mathcal{T}}: L^{*} \times L^{*} \longrightarrow L^{*}$ such that, for all $\mathbf{x}=\left(x_{1}, x_{2}\right), \mathbf{y}=\left(y_{1}, y_{2}\right) \in L^{*}$,

$$
\begin{aligned}
I_{\mathcal{T}}(\mathbf{x}, \mathbf{y}) & =\sup \left\{\mathbf{z} \mid \mathbf{z} \in L^{*}, \mathcal{T}(\mathbf{x}, \mathbf{z}) \leq{ }_{L^{*}} \mathbf{y}\right\} \\
& =\sup \left\{\left(z_{1}, z_{2}\right) \mid\left(z_{1}, z_{2}\right) \in L^{*}, \top\left(x_{1}, z_{1}\right) \leq y_{1} \text { and } S\left(x_{2}, z_{2}\right) \geq y_{2}\right\} .
\end{aligned}
$$

2. An intuitionistic fuzzy co-implication (for short, IF-co-implication) associated with an IF-tconorm, $\mathcal{J}=(S, \top)$, is a mapping $J_{\mathcal{J}}: L^{*} \times L^{*} \longrightarrow L^{*}$ such that, for all $\mathbf{x}=\left(x_{1}, x_{2}\right), \mathbf{y}=$ $\left(y_{1}, y_{2}\right) \in L^{*}$,

$$
\begin{aligned}
J_{\mathcal{J}}(\mathbf{x}, \mathbf{y}) & =\inf \left\{\mathbf{z} \mid \mathbf{z} \in L^{*}, \mathbf{y} \leq_{L^{*}} \mathcal{J}(\mathbf{x}, \mathbf{z})\right\} \\
& =\inf \left\{\left(z_{1}, z_{2}\right) \mid\left(z_{1}, z_{2}\right) \in L^{*}, y_{1} \leq \top\left(x_{1}, z_{1}\right) \text { and } y_{2} \geq S\left(x_{2}, z_{2}\right)\right\} .
\end{aligned}
$$

The following useful result relates IF-co-implication and IF-R-implication associated with an IF-t-conorm, $\mathcal{J}=(S, \top)$ and IF-t-norm, $\mathcal{T}=(\top, S)$, respectively to corresponding fuzzy co-implication, $J_{S}$ associated to $S$ and fuzzy R-implication, $I_{\top}$ associated to $\top$.

Lemma 1 (see [13]). For any $\mathbf{x}=\left(x_{1}, x_{2}\right), \mathbf{y}=\left(y_{1}, y_{2}\right) \in L^{*}$, we have

1. $J_{\mathcal{J}}(\mathbf{x}, \mathbf{y})=\left(J_{S}\left(x_{1}, y_{1}\right), \min \left(I_{\top}\left(x_{2}, y_{2}\right), 1-J_{S}\left(x_{1}, y_{1}\right)\right)\right)$.

2. $I_{\mathcal{T}}(\mathbf{x}, \mathbf{y})=\left(\min \left(I_{\top}\left(x_{1}, y_{1}\right), 1-J_{S}\left(x_{2}, y_{2}\right)\right), J_{S}\left(x_{2}, y_{2}\right)\right)$. 
The following are examples of t-representable IF-t-norms and IF-t-conorms [13].

Example 1. i. $\mathcal{T}_{M}=\left(\top_{M}, S_{M}\right)$ and $\mathcal{J}_{M}=\left(S_{M}, \top_{M}\right)$ are t-representable IF-t-norm and IF-tconorm respectively associated to $\top_{M}$ and $S_{M}$.

ii. $\mathcal{T}_{P}=\left(\top_{P}, S_{P}\right)$ and $\mathcal{J}_{P}=\left(S_{P}, \top_{P}\right)$ are $t$-representable IF-t-norm and IF-t-conorm respectively associated to $\top_{P}$ and $S_{P}$.

iii. $\mathcal{T}_{L}=\left(\top_{L}, S_{L}\right)$ and $\mathcal{J}_{L}=\left(S_{L}, \top_{L}\right)$ are t-representable IF-t-norm and IF-t-conorm respectively associated to $\top_{L}$ and $S_{L}$.

iv. Also, by verifying that $\top_{F}^{l}(a, b) \leq 1-S_{F}^{l}(1-a, 1-b)$ holds for all $a, b \in[0,1], l \in$ $(0,1) \cup(1,+\infty), \mathcal{T}_{F}^{l}=\left(\top_{F}^{l}, S_{F}^{l}\right)$ and $\mathcal{J}_{F}^{l}=\left(S_{F}^{l}, \top_{F}^{l}\right)$ are t-representable IF-t-norm and IF-t-conorm respectively associated to $\top_{F}^{l}$ and $S_{F}^{l}$ for all $l \in(0,1) \cup(1,+\infty)$.

Using Lemma 1 and Example 8 (see the Appendix), we construct the following examples of IF-R-implication and IF-co-implication associated with an IF-t-norm, $\mathcal{T}=(\top, S)$ and IF-tconorm, $\mathcal{J}=(S, \top)$.

Example 2. For all $\mathbf{x}=\left(x_{1}, x_{2}\right), \mathbf{y}=\left(y_{1}, y_{2}\right) \in L^{*}$,

1. The IF-R-implication associated with $\mathcal{T}_{M}=\left(\top_{M}, S_{M}\right)$ and the IF-co-implication associated with $\mathcal{J}_{M}=\left(S_{M}, \top_{M}\right)$ are respectively given by:

$$
I_{\mathcal{T}_{M}}(\mathbf{x}, \mathbf{y})=\left\{\begin{array}{l}
(1,0), \quad \text { if } \mathbf{x} \leq_{L^{*}} \mathbf{y}, \\
\left(\min \left(y_{1}, 1-y_{2}\right), y_{2}\right), \quad \text { if } \mathbf{x}>_{L^{*}} \mathbf{y} .
\end{array}\right.
$$

and

$$
J_{\mathcal{J}_{M}(\mathbf{x}, \mathbf{y})}=\left\{\begin{array}{l}
(0,1), \quad \text { if } \mathbf{x} \geq_{L^{*}} \mathbf{y}, \\
\left(y_{1}, \min \left(y_{2}, 1-y_{1}\right)\right), \quad \text { if } \mathbf{x}<_{L^{*}} \mathbf{y}
\end{array}\right.
$$

2. IF-R-implication associated with $\mathcal{T}_{P}=\left(\top_{P}, S_{P}\right)$ and IF-co-implication associated with $\mathcal{J}_{P}=\left(S_{P}, \top_{P}\right)$ are respectively given by:

$$
I_{\mathcal{T}_{P}}(\mathbf{x}, \mathbf{y})=\left\{\begin{array}{l}
(1,0), \quad \text { if } \mathbf{x} \leq_{L^{*}} \mathbf{y}, \\
\left(\min \left(\frac{y_{1}}{x_{1}}, \frac{1-y_{2}}{1-x_{2}}\right), \frac{y_{2}-x_{2}}{1-x_{2}}\right), \quad \text { if } \mathbf{x}>_{L^{*}} \mathbf{y}
\end{array}\right.
$$

and

$$
J_{\mathcal{J}_{P}(\mathbf{x}, \mathbf{y})}=\left\{\begin{array}{l}
(0,1), \quad \text { if } \mathbf{x} \geq_{L^{*}} \mathbf{y}, \\
\left(\frac{y_{1}-x_{1}}{1-x_{1}}, \min \left(\frac{y_{2}}{x_{2}}, \frac{1-y_{1}}{1-x_{1}}\right)\right), \quad \text { if } \mathbf{x}<_{L^{*}} \mathbf{y} .
\end{array}\right.
$$

3. The IF-R-implication associated with $\mathcal{T}_{L}=\left(\top_{L}, S_{L}\right)$ and the IF-co-implication associated with $\mathcal{J}_{L}=\left(S_{L}, \top_{L}\right)$ are respectively given by:

$$
I_{\mathcal{T}_{L}}(\mathbf{x}, \mathbf{y})=\left\{\begin{array}{l}
(1,0), \quad \text { if } \mathbf{x} \leq_{L^{*}} \mathbf{y}, \\
\left(\min \left(1-x_{1}+y_{1}, 1+x_{2}-y_{2}\right), y_{2}-x_{2}\right), \quad \text { if } \mathbf{x}>_{L^{*}} \mathbf{y} .
\end{array}\right.
$$


and

$$
J_{\mathcal{J}_{L}(\mathbf{x}, \mathbf{y})}=\left\{\begin{array}{l}
(0,1), \quad \text { if } \mathbf{x} \geq_{L^{*}} \mathbf{y}, \\
\left(y_{1}-x_{1}, \min \left(1-x_{2}+y_{2}, 1+x_{1}-y_{1}\right)\right), \quad \text { if } \mathbf{x}<_{L^{*}} \mathbf{y} .
\end{array}\right.
$$

4. The IF-R-implication associated with $\mathcal{T}_{F}^{l}=\left(T_{F}^{l}, S_{F}^{l}\right)$ and the IF-co-implication associated with $\mathcal{J}_{F}^{l}=\left(S_{F}^{l}, \top_{F}^{l}\right)$ for all $l \in(0,1) \cup(1,+\infty)$ are respectively given by:

$$
I_{\mathcal{T}_{F}^{l}}(\mathbf{x}, \mathbf{y})=\left\{\begin{array}{l}
(1,0), \quad \text { if } \mathbf{x} \leq_{L^{*}} \mathbf{y}, \\
\left(\min \left(\log _{l}\left(1+\frac{(l-1)\left(l^{y_{1}}-1\right)}{l^{x}-1}\right), \log _{l}\left(1+\frac{(l-1)\left(l^{1-y_{2}}-1\right)}{l^{1-x_{2}}-1}\right)\right),\right. \\
\left.1-\log _{l}\left(1+\frac{(l-1)\left(l^{1-y_{2}}-1\right)}{l^{1-x_{2}}-1}\right)\right), \quad \text { if } \mathbf{x}>_{L^{*}} \mathbf{y},
\end{array}\right.
$$

and

$$
J_{\mathcal{J}_{F}^{l}(\mathbf{x}, \mathbf{y})}=\left\{\begin{array}{l}
(0,1), \quad \text { if } \mathbf{x} \geq_{L^{*}} \mathbf{y}, \\
\left(1-\log _{l}\left(1+\frac{(l-1)\left(l^{1-y_{1}}-1\right)}{l^{1-x_{1}-1}}\right),\right. \\
\left.\min \left(\log _{l}\left(1+\frac{(l-1)\left(l^{y_{2}}-1\right)}{l^{x_{2}-1}}\right), \log _{l}\left(1+\frac{(l-1)\left(l^{1-y_{2}}-1\right)}{l^{1-x_{2}-1}}\right)\right)\right), \quad \text { if } \mathbf{x}<_{L^{*}} \mathbf{y} .
\end{array}\right.
$$

We end this Subsection by recalling inclusion and some operations on intuitionistic fuzzy sets.

Definition 6 (Intuitionistic Fuzzy Operations [2,7,9,11,12]). Let $A$ and $B$ be any two intuitionistic fuzzy sets defined on $X$. Inclusion and the following operations are defined by associated membership and non-membership functions as follows:

i. Inclusion: $A \subseteq B$ if $\forall x \in X, \mu_{A}(x) \leq \mu_{B}(x) \quad$ and $\quad \nu_{A}(x) \geq \nu_{B}(x)$;

ii. Intersection: $A \cap B$ is defined by: $\forall x \in X,\left(\mu_{A \cap B}(x), \nu_{A \cap B}(x)\right)=\left(\mu_{A}(x) \wedge \mu_{B}(x), \nu_{A}(x) \vee \nu_{B}(x)\right)$;

iii. Union: $A \cup B$ is defined by: $\forall x \in X,\left(\mu_{A \cup B}(x), \nu_{A \cup B}(x)\right)=\left(\mu_{A}(x) \vee \mu_{B}(x), \nu_{A}(x) \wedge \nu_{B}(x)\right)$;

iv. Complement: $A^{c}$ is defined by: $\forall x \in X,\left(\mu_{A^{c}}(x), \nu_{A^{c}}(x)\right)=\left(\nu_{A}(x), \mu_{A}(x)\right)$;

v. Difference: $A-B$ is defined by: $\forall x \in X,\left(\mu_{A-B}(x), \nu_{A-B}(x)\right)=\left(\mu_{A}(x) \wedge \nu_{B}(x), \nu_{A}(x) \vee \mu_{B}(x)\right)$;

vi. Symmetric Difference: $A \triangle B$ is defined by: $\forall x \in X,\left(\mu_{A \triangle B}(x), \nu_{A \triangle B}(x)\right)=$ $\left(\min \left\{\mu_{A}(x) \vee \mu_{B}(x), \nu_{A}(x) \vee \nu_{B}(x)\right\}, \max \left\{\nu_{A}(x) \wedge \nu_{B}(x), \mu_{A}(x) \wedge \mu_{B}(x)\right\}\right)$.

In the next Subsection, we recall the difference and symmetric difference operations for fuzzy sets, some examples and their properties as proposed by Fono et al. [10].

\subsection{Difference and symmetric difference of fuzzy sets based on fuzzy implications}

Definition 7 (Difference and Symmetric Difference Operations for Fuzzy Sets [10]). a. Let $M, N$ be any two fuzzy sets defined on $X$ and $i \in\{1,2,3,4\}$. The fuzzy difference of type $i$ associated to $\top$ of $M$ and $N$ is the fuzzy set of $X$ denoted by $M \frac{i}{T} N$ and defined by:

$$
\mu_{M \underset{\top}{i} N}(x)=\overline{I_{\top}^{i}}\left(\mu_{M}(x), \mu_{N}(x)\right)=1-I_{\top}^{i}\left(\mu_{M}(x), \mu_{N}(x)\right), \quad \text { for all } x \in X .
$$


b. The fuzzy symmetric difference of type $i \in\{1,2\}$ associated to $\top$ of $M$ and $N$ is the fuzzy set of $X$ denoted by $M \stackrel{i}{\triangle} N$ and defined for all $x \in X$ by:

$$
\mu_{M_{\uparrow}^{i} N}(x)=\mu_{M \cup N \underset{\top}{-} M \cap N}(x)= \begin{cases}1-I_{\top}^{1}\left(\mu_{M}(x) \vee \mu_{N}(x), \mu_{M}(x) \wedge \mu_{N}(x)\right), & \text { if } i=1 \\ 1-I_{\top}^{2}\left(\mu_{M}(x) \vee \mu_{N}(x), \mu_{M}(x) \wedge \mu_{N}(x)\right), & \text { if } i=2 .\end{cases}
$$

We recall the examples of these operations for fuzzy sets of type 1 and 2 associated to the usual three fuzzy t-norms in what follows.

Example 3. For any fuzzy sets $M$ and $N$ defined on $X$,

1. Examples of fuzzy difference operations

(a) The difference operation associated with $\top_{M}$ is given by, for all $x \in X$

$$
\begin{gathered}
\mu_{M_{T_{M}}^{-1} N}(x)=\left\{\begin{array}{l}
0, \quad \text { if } \mu_{M}(x) \leq \mu_{N}(x), \\
1-\mu_{N}(x), \quad \text { if } \mu_{M}(x)>\mu_{N}(x),
\end{array}\right. \\
\mu_{M_{T_{M}} \frac{2}{-} N}(x)= \begin{cases}0, & \text { if } \mu_{M}(x) \leq \mu_{N}(x), \\
\mu_{M}(x), & \text { if } \mu_{M}(x)>\mu_{N}(x)\end{cases}
\end{gathered}
$$

(b) The difference operation associated with $\top_{P}$ is given by, for all $x \in X$

$$
\begin{gathered}
\mu_{M_{T_{P}}^{\frac{1}{T_{P}} N}}(x)= \begin{cases}0, & \text { if } \mu_{M}(x) \leq \mu_{N}(x), \\
1-\frac{\mu_{N}(x)}{\mu_{M}(x)}, & \text { if } \mu_{M}(x)>\mu_{N}(x),\end{cases} \\
\mu_{M_{T_{P}}^{\frac{2}{T_{P}} N}}(x)= \begin{cases}0, & \text { if } \mu_{M}(x) \leq \mu_{N}(x), \\
\frac{\mu_{M}(x)-\mu_{N}(x)}{1-\mu_{N}(x)}, & \text { if } \mu_{M}(x)>\mu_{N}(x) .\end{cases}
\end{gathered}
$$

(c) The difference operation associated with $\top_{L}$ is given by, for all $x \in X$ and $i \in\{1,2\}$,

$$
\mu_{M_{\mathrm{T}_{L}}^{\stackrel{i}{-} N}}(x)=\left\{\begin{array}{l}
0, \quad \text { if } \mu_{M}(x) \leq \mu_{N}(x) \\
\mu_{M}(x)-\mu_{N}(x), \quad \text { if } \mu_{M}(x)>\mu_{N}(x)
\end{array}\right.
$$

2. Examples of fuzzy symmetric difference operations

(a) The symmetric difference operation associated with $\top_{M}$ is given by, for all $x \in X$

$$
\begin{aligned}
& \mu_{M_{T_{M} \Delta}^{\Delta} \Delta}(x)=\left\{\begin{array}{l}
0, \quad \text { if } \mu_{M}(x)=\mu_{N}(x), \\
\max \left(1-\mu_{M}(x), 1-\mu_{N}(x)\right), \quad \text { if } \mu_{M}(x) \neq \mu_{N}(x),
\end{array}\right. \\
& \mu_{M_{T_{M}}^{\Delta}{ }_{M}^{2} N}(x)=\left\{\begin{array}{l}
0, \quad \text { if } \mu_{M}(x)=\mu_{N}(x), \\
\max \left(\mu_{M}(x), \mu_{N}(x)\right), \quad \text { if } \mu_{M}(x) \neq \mu_{N}(x) .
\end{array}\right.
\end{aligned}
$$


(b) The symmetric difference operation associated with $\top_{P}$ is given by, for all $x \in X$

$$
\begin{aligned}
& \mu_{M_{T_{P}}^{\Delta} N}(x)=\left\{\begin{array}{l}
0, \quad \text { if } \mu_{M}(x)=\mu_{N}(x)=0, \\
\frac{\left|\mu_{M}(x)-\mu_{N}(x)\right|}{\max \left\{\mu_{M}(x), \mu_{N}(x)\right\}}, \quad \text { if } \mu_{M}(x) \neq 0, \text { or } \mu_{N}(x) \neq 0,
\end{array}\right. \\
& \mu_{M_{T_{P}}^{\Delta} N}^{2}(x)=\left\{\begin{array}{l}
0, \quad \text { if } \mu_{M}(x)=\mu_{N}(x)=1, \\
\frac{\left|\mu_{M}(x)-\mu_{N}(x)\right|}{1-\min \left\{\mu_{M}(x), \mu_{N}(x)\right\}}, \quad \text { if } \mu_{M}(x) \wedge \mu_{N}(x)<1 .
\end{array}\right.
\end{aligned}
$$

(c) The symmetric difference operation associated with $\top_{L}$ is given by, for all $x \in X$ and $i \in\{1,2\}$,

$$
\mu_{M_{T_{L}}^{\Delta} N}^{i}(x)=\left|\mu_{M}(x)-\mu_{N}(x)\right| .
$$

Fono et al. [10] have also proved that the difference and symmetric difference operations for fuzzy sets of type 1 and 2 associated to any continuous t-norm $\top$ so defined preserve the properties of the classical difference and symmetric difference operation for crisp sets. We recall these results as follows:

Proposition 1. Let $i \in\{1,2\}$ and $M, M^{\prime}, N$ be any arbitrary fuzzy sets on $X$. The following properties hold [10]:

1. Properties of fuzzy difference operation;

(a) if $M \subseteq N$, then $M \frac{i}{\mathrm{~T}} N=\emptyset$, (b) if $M \subseteq M^{\prime}$, then $M \frac{i}{\mathrm{~T}} N \subseteq M^{\prime} \frac{i}{\mathrm{~T}} N$, (c) if $M \subseteq M^{\prime}$, then $N \frac{i}{\mathrm{~T}} M^{\prime} \subseteq N \frac{i}{\mathrm{~T}} M,(d)\left(M \frac{i}{\mathrm{~T}} N\right) \cap\left(N \frac{i}{\mathrm{~T}} M\right)=\emptyset$ and $(e) M \frac{i}{\mathrm{~T}} N=M \frac{i}{\mathrm{~T}}(M \cap N)$.

2. Properties of fuzzy symmetric difference operation;
(a) $M \stackrel{i}{\triangle} N=\left(M \frac{i}{\mathrm{~T}} N\right) \cup\left(N \frac{i}{\mathrm{~T}} M\right)$,
(b) if $M \subseteq N$, then $M \stackrel{i}{\triangle} N=N \frac{i}{\top} M$ and (c) $M \stackrel{i}{\triangle} M=\emptyset$.

The following result shows that, the fuzzy complement of fuzzy sets associated with any continuous t-norm $T$ so defined, preserve the property of the classical complement for crisp sets.

Corollary 1. Let $\top$ be any continuous t-norm, $A$ be a fuzzy set on $X$, and $A^{c}$ be the fuzzy complement of $A$ associated with $\top$.

Then $A^{c}=X-\top A$.

Proof. Let $x \in X$. From Definition 12 (see the Appendix), it is sufficient to show that: $\mu_{X{ }_{-\top} A}(x)=$ $1-\mu_{A}(x)$.

Since $\mu_{X}(x)=1$ and $I_{\top}(1, a)=a$ (see Proposition 9 in the Appendix) for all $a \in[0 ; 1]$, from Definition 7 ,

$\mu_{X-\top A}(x)=1-I_{\top}\left(\mu_{X}(x), \mu_{A}(x)\right)=1-\mu_{A}(x)$.

In the following Section, we introduce new operations for intuitionistic fuzzy sets and establish some of their properties. 


\section{New operations for intuitionistic fuzzy sets: Difference and symmetric difference}

\subsection{Definitions and properties of difference operations}

Let $I_{\mathcal{T}}=\left({ }_{1} I_{\mathcal{T},{ }_{2}} I_{\mathcal{T}}\right)$ be an IF-R-implication operator. We define the negation of $I_{\mathcal{T}}$ as $\mathcal{N}\left(I_{\mathcal{T}}\right)=$ $\left({ }_{2} I_{\mathcal{T}, 1} I_{\mathcal{T}}\right)$. In particular, using Lemma 1 we define the negation of IF-R-implication as $\forall \mathbf{x}=$ $\left(x_{1}, x_{2}\right), \mathbf{y}=\left(y_{1}, y_{2}\right) \in L^{*}, \mathcal{N}\left(I_{\mathcal{T}}(\mathbf{x}, \mathbf{y})\right)=\left(J_{S}\left(x_{2}, y_{2}\right), \min \left\{I_{\top}\left(x_{1}, y_{1}\right), 1-J_{S}\left(x_{2}, y_{2}\right)\right\}\right)$.

Definition 8. Let $A, B$ be any two intuitionistic fuzzy sets defined on $X$. The intuitionistic fuzzy difference associated to $\mathcal{T}$ of $A$ and $B$ is the intuitionistic fuzzy set on $X$ denoted by $A-\mathcal{T} B$ and defined by the membership and non-membership degrees as follows:

For all $x \in X$,

$$
\begin{aligned}
\left(\mu_{A-\mathcal{T} B}(x), \nu_{A-\mathcal{T} B}(x)\right) & =\mathcal{N}\left(I_{\mathcal{T}}\left(\left(\mu_{A}(x), \nu_{A}(x)\right),\left(\mu_{B}(x), \nu_{B}(x)\right)\right)\right) \\
& =\left(J_{S}\left(\nu_{A}(x), \nu_{B}(x)\right), \min \left\{I_{\top}\left(\mu_{A}(x), \mu_{B}(x)\right), 1-J_{S}\left(\nu_{A}(x), \nu_{B}(x)\right)\right\}\right) .
\end{aligned}
$$

The following are typical examples of difference operations associated with the three usual and well-known $\mathcal{T}$.

Example 4. For any intuitionistic fuzzy sets $A$ and $B$ defined on $X$,

1. The difference operation associated with $\mathcal{T}_{M}$ is given by, for all $x \in X$

$$
\left(\mu_{A-\tau_{M} B}(x), \nu_{A-\tau_{M} B}(x)\right)=\left\{\begin{array}{l}
(0,1), \quad \text { if }\left(\mu_{A}(x), \nu_{A}(x)\right) \leq_{L^{*}}\left(\mu_{B}(x), \nu_{B}(x)\right), \\
\left(\nu_{B}(x), \min \left\{\mu_{B}(x), 1-\nu_{B}(x)\right\}\right) \\
\text { if }\left(\mu_{A}(x), \nu_{A}(x)\right)>_{L^{*}}\left(\mu_{B}(x), \nu_{B}(x)\right) .
\end{array}\right.
$$

2. The difference operation associated with $\mathcal{T}_{P}$ is given by, for all $x \in X$

$$
\left(\mu_{A-\mathcal{T}_{P} B}(x), \nu_{A-\tau_{P} B}(x)\right)=\left\{\begin{array}{l}
(0,1), \quad \text { if }\left(\mu_{A}(x), \nu_{A}(x)\right) \leq_{L^{*}}\left(\mu_{B}(x), \nu_{B}(x)\right) \\
\left(\frac{\nu_{B}(x)-\nu_{A}(x)}{1-\nu_{A}(x)}, \min \left\{\frac{\mu_{B}(x)}{\mu_{A}(x)}, \frac{1-\nu_{B}(x)}{1-\nu_{A}(x)}\right\}\right) \\
\text { if }\left(\mu_{A}(x), \nu_{A}(x)\right)>_{L^{*}}\left(\mu_{B}(x), \nu_{B}(x)\right) .
\end{array}\right.
$$

3. The difference operation associated with $\mathcal{T}_{L}$ is given by, for all $x \in X$

$$
\left(\mu_{A-\tau_{L} B}(x), \nu_{A-\tau_{L} B}(x)\right)=\left\{\begin{array}{l}
(0,1), \quad \text { if }\left(\mu_{A}(x), \nu_{A}(x)\right) \leq_{L^{*}}\left(\mu_{B}(x), \nu_{B}(x)\right) \\
\left(\nu_{B}(x)-\nu_{A}(x), \min \left\{1-\mu_{A}(x)+\mu_{B}(x), 1+\nu_{A}(x)\right.\right. \\
\left.\left.-\nu_{B}(x)\right\}\right), \text { if }\left(\mu_{A}(x), \nu_{A}(x)\right)>_{L^{*}}\left(\mu_{B}(x), \nu_{B}(x)\right) .
\end{array}\right.
$$

In the following results, we establish four classical properties for difference operation which are satisfied by the new intuitionistic fuzzy difference operation.

Proposition 2 (Properties of Intuitionistic Fuzzy Difference Operation). Let A, B, C be intuitionistic fuzzy sets on $X$. The following properties for intuitionistic fuzzy difference operations hold: 
1. if $A \subseteq B$, then $A-\mathcal{T} B=\emptyset$;

2. if $A \subseteq B$, then $A-{ }_{\mathcal{T}} C \subseteq B-{ }_{\mathcal{T}} C$;

3. if $A \subseteq B$, then $C-\mathcal{T} B \subseteq C-\mathcal{T} A$;

4. $A-\mathcal{T} B=A-\mathcal{T}(A \cap B)$.

Proof. By Proposition 9 and Definition 8, we establish the results for all $x \in X$ as follows:

1. Assume that, $A \subseteq B$, then $\mu_{A}(x) \leq \mu_{B}(x)$ and $\nu_{A}(x) \geq \nu_{B}(x)$.

Since $J_{S}\left(\nu_{A}(x), \nu_{B}(x)\right)=0$, whenever $\nu_{A}(x) \geq \nu_{B}(x)$, and $I_{\top}\left(\mu_{A}(x), \mu_{B}(x)\right)=1$, whenever $\mu_{A}(x) \leq \mu_{B}(x)$ then by Definition 8 , we have $\left(\mu_{A-\mathcal{T} B}(x), \nu_{A-\tau B}(x)\right)=(0,1)$ and the result follows.

2. Assume that $A \subseteq B$, then $\mu_{A}(x) \leq \mu_{B}(x)$ and $\nu_{A}(x) \geq \nu_{B}(x)$.

$$
\begin{aligned}
& \left(\mu_{A-\mathcal{T} C}(x), \nu_{A-\mathcal{T} C}(x)\right)=\left(J_{S}\left(\nu_{A}(x), \nu_{C}(x)\right), \min \left\{I_{\top}\left(\mu_{A}(x), \mu_{C}(x)\right), 1-J_{S}\left(\nu_{A}(x), \nu_{C}(x)\right)\right\}\right), \\
& \left(\mu_{B-\mathcal{T} C}(x), \nu_{B-\mathcal{T} C}(x)\right)=\left(J_{S}\left(\nu_{B}(x), \nu_{C}(x)\right), \min \left\{I_{\top}\left(\mu_{B}(x), \mu_{C}(x)\right), 1-J_{S}\left(\nu_{B}(x), \nu_{C}(x)\right)\right\}\right) .
\end{aligned}
$$

Since $\nu_{B}(x) \leq \nu_{A}(x)$, then from Proposition $9 J_{S}\left(\nu_{A}(x), \nu_{C}(x)\right) \leq J_{S}\left(\nu_{B}(x), \nu_{C}(x)\right)$.

So, $\mu_{A-\mathcal{T} C}(x) \leq \mu_{B-\mathcal{T} C}(x)$.

For the non-membership degree, there are four possibilities:

Case i: $\nu_{A-\mathcal{T} C}(x)=I_{\top}\left(\mu_{A}(x), \mu_{C}(x)\right)$ and $\nu_{B-{ }_{\mathcal{T}} C}(x)=I_{\top}\left(\mu_{B}(x), \mu_{C}(x)\right)$.

Since $\mu_{A}(x) \leq \mu_{B}(x)$, then $I_{\top}\left(\mu_{A}(x), \mu_{C}(x)\right) \geq I_{\top}\left(\mu_{B}(x), \mu_{C}(x)\right)$ and we have $\nu_{A-\mathcal{T} C}(x) \geq \nu_{B-\mathcal{T} C}(x)$.

Case ii: $\nu_{A-{ }_{\mathcal{T}} C}(x)=1-J_{S}\left(\nu_{A}(x), \nu_{C}(x)\right)$ and $\nu_{B-{ }_{\mathcal{T}} C}(x)=1-J_{S}\left(\nu_{B}(x), \nu_{C}(x)\right)$.

Since $\nu_{B}(x) \leq \nu_{A}(x)$ then, $J_{S}\left(\nu_{A}(x), \nu_{C}(x)\right) \leq J_{S}\left(\nu_{B}(x), \nu_{C}(x)\right)$, then we have $\nu_{A-\mathcal{T} C}(x) \geq \nu_{B-\mathcal{T} C}(x)$.

Case iii: $\nu_{A-{ }_{\mathcal{T}} C}(x)=I_{\top}\left(\mu_{A}(x), \mu_{C}(x)\right)$ and $\nu_{B-{ }_{\mathcal{T}} C}(x)=1-J_{S}\left(\nu_{B}(x), \nu_{C}(x)\right)$.

Since $\mu_{A}(x) \leq \mu_{B}(x)$, then

$$
I_{\top}\left(\mu_{A}(x), \mu_{C}(x)\right) \geq I_{\top}\left(\mu_{B}(x), \mu_{C}(x)\right) \geq 1-J_{S}\left(\nu_{B}(x), \nu_{C}(x)\right) .
$$

So, $\nu_{A-\mathcal{T} C}(x) \geq \nu_{B-\mathcal{T} C}(x)$.

Case iv: $\nu_{A-{ }_{\mathcal{T}} C}(x)=1-J_{S}\left(\nu_{A}(x), \nu_{C}(x)\right)$ and $\nu_{B-\mathcal{T} C}(x)=I_{\top}\left(\mu_{B}(x), \mu_{C}(x)\right)$.

Since $\nu_{A}(x) \geq \nu_{B}(x)$, then

$$
1-J_{S}\left(\nu_{A}(x), \nu_{C}(x)\right) \geq 1-J_{S}\left(\nu_{B}(x), \nu_{C}(x)\right) \geq I_{\top}\left(\mu_{B}(x), \mu_{C}(x)\right) .
$$

So, $\nu_{A-\mathcal{T} C}(x) \geq \nu_{B-\mathcal{T} C}(x)$.

Thus for all $x \in X, \mu_{A-\mathcal{T} C}(x) \leq \mu_{B-\mathcal{T} C}(x)$ and $\nu_{A-_{\mathcal{T}} C}(x) \geq \nu_{B-\mathcal{T} C}(x)$.

So, $A-{ }_{\mathcal{T}} C \subseteq B-{ }_{\mathcal{T}} C$. 
3. Assume that $A \subseteq B$ then, $\mu_{A}(x) \leq \mu_{B}(x)$ and $\nu_{A}(x) \geq \nu_{B}(x)$.

$\left(\mu_{C-\mathcal{T}_{B}}(x), \nu_{C-\mathcal{T} B}(x)\right)=\left(J_{S}\left(\nu_{C}(x), \nu_{B}(x)\right), \min \left\{I_{\top}\left(\mu_{C}(x), \mu_{B}(x)\right), 1-J_{S}\left(\nu_{C}(x), \nu_{B}(x)\right)\right\}\right)$,

$\left(\mu_{C-\mathcal{T} A}(x), \nu_{C-\mathcal{T} A}(x)\right)=\left(J_{S}\left(\nu_{C}(x), \nu_{A}(x)\right), \min \left\{I_{\top}\left(\mu_{C}(x), \mu_{A}(x)\right), 1-J_{S}\left(\nu_{C}(x), \nu_{A}(x)\right)\right\}\right)$.

Since $\nu_{B}(x) \leq \nu_{A}(x)$, then $J_{S}\left(\nu_{C}(x), \nu_{B}(x)\right) \leq J_{S}\left(\nu_{C}(x), \nu_{A}(x)\right)$. So, $\mu_{C-\mathcal{T} B}(x) \leq$ $\mu_{C-\mathcal{T} A}(x)$.

For the non-membership degree, there are four possibilities:

Case i: $\nu_{C-\mathcal{T} B}(x)=I_{\top}\left(\mu_{C}(x), \mu_{B}(x)\right)$ and $\nu_{C{ }_{-\mathcal{T}} A}(x)=I_{\top}\left(\mu_{C}(x), \mu_{A}(x)\right)$.

Since $\mu_{A}(x) \leq \mu_{B}(x)$, then $I_{\top}\left(\mu_{C}(x), \mu_{B}(x)\right) \geq I_{\top}\left(\mu_{C}(x), \mu_{A}(x)\right)$ and we have $\nu_{C-\mathcal{T} B}(x) \geq \nu_{C-\mathcal{T} A}(x)$.

Case ii: $\nu_{C-\mathcal{T} B}(x)=1-J_{S}\left(\nu_{C}(x), \nu_{B}(x)\right)$ and $\nu_{C-\mathcal{T} A}(x)=1-J_{S}\left(\nu_{C}(x), \nu_{A}(x)\right)$.

Since $\nu_{B}(x) \leq \nu_{A}(x)$ then $J_{S}\left(\nu_{C}(x), \nu_{B}(x)\right) \leq J_{S}\left(\nu_{C}(x), \nu_{A}(x)\right)$, then we have $\nu_{C-\mathcal{T} B}(x) \geq \nu_{C-\mathcal{T} A}(x)$.

Case iii: $\nu_{C-\mathcal{T} B}(x)=I_{\top}\left(\mu_{C}(x), \mu_{B}(x)\right)$ and $\nu_{C-\mathcal{T} A}(x)=1-J_{S}\left(\nu_{C}(x), \nu_{A}(x)\right)$. Since $\mu_{A}(x) \leq \mu_{B}(x)$, then

$$
I_{\top}\left(\mu_{C}(x), \mu_{B}(x)\right) \geq I_{\top}\left(\mu_{C}(x), \mu_{A}(x)\right) \geq 1-J_{S}\left(\nu_{C}(x), \nu_{A}(x)\right) .
$$

So, $\nu_{C-\mathcal{T} B}(x) \geq \nu_{C-\mathcal{T} A}(x)$.

Case iv: $\nu_{C-\mathcal{T} B}(x)=1-J_{S}\left(\nu_{C}(x), \nu_{B}(x)\right)$ and $\nu_{C-\mathcal{T} A}(x)=I_{\top}\left(\mu_{C}(x), \mu_{A}(x)\right)$.

Since $\nu_{A}(x) \geq \nu_{B}(x)$, then

$$
1-J_{S}\left(\nu_{C}(x), \nu_{B}(x)\right) \geq 1-J_{S}\left(\nu_{C}(x), \nu_{A}(x)\right) \geq I_{\top}\left(\mu_{C}(x), \mu_{A}(x)\right) .
$$

So, $\nu_{C-\mathcal{T} B}(x) \geq \nu_{C-\mathcal{T} A}(x)$.

Thus for all $x \in X, \mu_{C-\mathcal{T} B}(x) \leq \mu_{C-\mathcal{T} A}(x)$ and $\nu_{C-\mathcal{T} B}(x) \geq \nu_{C-\mathcal{T} A}(x)$.

So, $C-{ }_{\mathcal{T}} B \subseteq C-\mathcal{T} A$.

\section{From Definition 8 we have,}

$$
\begin{gathered}
\mu_{A-\mathcal{T}(A \cap B)}(x)=J_{S}\left(\nu_{A}(x), \nu_{A \cap B}(x)\right)=J_{S}\left(\nu_{A}(x), \max \left\{\nu_{A}(x), \nu_{B}(x)\right\}\right), \\
\nu_{A-\mathcal{T}(A \cap B)}(x)=\min \left\{I_{\top}\left(\mu_{A}(x), \mu_{A \cap B}(x)\right), 1-J_{S}\left(\nu_{A}(x), \nu_{A \cap B}(x)\right)\right\} \\
=\min \left\{I_{\top}\left(\mu_{A}(x), \min \left\{\mu_{A}(x), \mu_{B}(x)\right\}\right), 1-J_{S}\left(\nu_{A}(x), \max \left\{\nu_{A}(x), \nu_{B}(x)\right\}\right)\right\}, \\
\mu_{A-\mathcal{T} B}(x)=J_{S}\left(\nu_{A}(x), \nu_{B}(x)\right), \\
\nu_{A-\mathcal{T} B}(x)=\min \left\{I_{\top}\left(\mu_{A}(x), \mu_{B}(x)\right), 1-J_{S}\left(\nu_{A}(x), \nu_{B}(x)\right)\right\} .
\end{gathered}
$$

Claim:

We claim $\mu_{A-\mathcal{T}(A \cap B)}(x)=\mu_{A-\mathcal{T} B}(x)$ and $\nu_{A-\mathcal{T}(A \cap B)}(x)=\nu_{A-\mathcal{T} B}(x)$ for all $x \in X$. 
We note the following properties:

$J_{S}\left(\nu_{A}(x), \nu_{B}(x)\right)=0$, whenever $\nu_{A}(x) \geq \nu_{B}(x) ; I_{\top}\left(\mu_{A}(x), \mu_{B}(x)\right)=1$, whenever $\mu_{A}(x) \leq \mu_{B}(x) ; J_{S}\left(\nu_{A}(x), \nu_{A}(x)\right)=0$ and $I_{\top}\left(\mu_{A}(x), \mu_{A}(x)\right)=1$.

Then consider the following cases:

Case i: If $\mu_{A}(x) \leq \mu_{B}(x)$ and $\nu_{A}(x) \leq \nu_{B}(x)$, then by Equations (4)-(7), we have

$$
\begin{aligned}
\mu_{A-\mathcal{T}(A \cap B)}(x) & =J_{S}\left(\nu_{A}(x), \nu_{B}(x)\right)=\mu_{A-\mathcal{T} B}(x), \quad \text { and } \\
\nu_{A-\mathcal{T}(A \cap B)}(x) & =\min \left\{I_{\top}\left(\mu_{A}(x), \mu_{A}(x)\right), 1-J_{S}\left(\nu_{A}(x), \nu_{B}(x)\right)\right\} \\
& =\min \left\{1,1-J_{S}\left(\nu_{A}(x), \nu_{B}(x)\right)\right\} \\
& =\min \left\{I_{\top}\left(\mu_{A}(x), \mu_{B}(x)\right), 1-J_{S}\left(\nu_{A}(x), \nu_{B}(x)\right)\right\}=\nu_{A-\mathcal{T} B}(x) .
\end{aligned}
$$

Case ii: If $\mu_{A}(x) \leq \mu_{B}(x)$ and $\nu_{A}(x) \geq \nu_{B}(x)$, then by Equations (4)-(7), we have

$$
\begin{aligned}
\mu_{A-\mathcal{T}(A \cap B)}(x) & =J_{S}\left(\nu_{A}(x), \nu_{A}(x)\right)=0 \\
& =J_{S}\left(\nu_{A}(x), \nu_{B}(x)\right)=\mu_{A-\mathcal{T} B}(x), \quad \text { and } \\
\nu_{A-\mathcal{T}(A \cap B)}(x) & =\min \left\{I_{\top}\left(\mu_{A}(x), \mu_{A}(x)\right), 1-J_{S}\left(\nu_{A}(x), \nu_{A}(x)\right)\right\}=\min \{1,1-0\} \\
& =\min \left\{I_{\top}\left(\mu_{A}(x), \mu_{B}(x)\right), 1-J_{S}\left(\nu_{A}(x), \nu_{B}(x)\right)\right\}=\nu_{A-\mathcal{T} B}(x) .
\end{aligned}
$$

Case iii: If $\mu_{A}(x) \geq \mu_{B}(x)$ and $\nu_{A}(x) \leq \nu_{B}(x)$, then by Equations (4)-(7), we have

$$
\begin{aligned}
& \mu_{A-\mathcal{T}(A \cap B)}(x)=J_{S}\left(\nu_{A}(x), \nu_{B}(x)\right)=\mu_{A-\mathcal{T} B}(x), \quad \text { and } \\
& \nu_{A-\mathcal{T}(A \cap B)}(x)=\min \left\{I_{\top}\left(\mu_{A}(x), \mu_{B}(x)\right), 1-J_{S}\left(\nu_{A}(x), \nu_{B}(x)\right)\right\}=\nu_{A-\mathcal{T} B}(x) .
\end{aligned}
$$

Case iv: If $\mu_{A}(x) \geq \mu_{B}(x)$ and $\nu_{A}(x) \geq \nu_{B}(x)$, then by Equations (4)-(7), we have

$$
\begin{aligned}
\mu_{A-\mathcal{T}(A \cap B)}(x) & =J_{S}\left(\nu_{A}(x), \nu_{A}(x)\right)=0 \\
& =J_{S}\left(\nu_{A}(x), \nu_{B}(x)\right)=\mu_{A-\mathcal{T} B}(x), \quad \text { and } \\
\nu_{A-\mathcal{T}(A \cap B)}(x) & =\min \left\{I_{\top}\left(\mu_{A}(x), \mu_{B}(x)\right), 1-J_{S}\left(\nu_{A}(x), \nu_{A}(x)\right)\right\} \\
& =\min \left\{I_{\top}\left(\mu_{A}(x), \mu_{B}(x)\right), 1-0\right\} \\
& =\min \left\{I_{\top}\left(\mu_{A}(x), \mu_{B}(x)\right), 1-J_{S}\left(\nu_{A}(x), \nu_{B}(x)\right)\right\}=\nu_{A-\mathcal{T} B}(x) .
\end{aligned}
$$

Hence, $\left(\mu_{A-\mathcal{T}(A \cap B)}(x), \nu_{A-\mathcal{T}(A \cap B)}(x)\right)=\left(\mu_{A-\mathcal{T} B}(x), \nu_{A-\mathcal{T} B}(x)\right)$ for all $x \in X$, and the result follows.

The following result shows that, the intuitionistic fuzzy complement of fuzzy sets associated with a t-representable of an IF-t-norm $\mathcal{T}=(\top, S)$ so defined, preserve the property of the classical complement for crisp sets.

Corollary 2. Let $A$ be any intuitionistic fuzzy set of $X . A_{\mathcal{T}}^{c}$ be the intuitionistic fuzzy complement of $A$. Then $A_{\mathcal{T}}^{c}=X-_{\mathcal{T}} A$. 
Proof. Let $x \in X$. Since $\left(\mu_{X}(x), \nu_{X}(x)\right)=(1,0)$, then from Definition 8 ,

$$
\begin{aligned}
\left(\mu_{X-\mathcal{T}_{A}}(x), \nu_{X-\mathcal{T} A}(x)\right) & =\left(J_{S}\left(0, \nu_{A}(x)\right), \min \left\{I_{\top}\left(1, \mu_{A}(x)\right), 1-J_{S}\left(0, \nu_{A}(x)\right)\right\}\right), \\
& =\left(\nu_{A}(x), \min \left\{\mu_{A}(x), 1-\nu_{A}(x)\right\}\right), \quad \text { (recalling Prop. 9(1)), } \\
& =\left(\nu_{A}(x), \mu_{A}(x)\right), \quad \text { since } \mu_{A}(x) \leq 1-\nu_{A}(x) .
\end{aligned}
$$

From Definition 6, the result follows.

The following result establishes a property of the new difference operation.

Proposition 3. Let $A$ and $B$ be any intuitionistic fuzzy sets on $X$.

1. Then $(A-\mathcal{T} B) \cap_{\mathcal{T}}(B-\mathcal{T} A)$ is an intuitionistic fuzzy set with membership function, $\mu_{(A-\mathcal{T} B) \cap_{\mathcal{T}}(B-\mathcal{T} A)}(x)=0, \forall x \in X$ and non-membership function defined by: for all $x \in X$,

$$
\nu_{(A-\mathcal{T} B) \cap_{\mathcal{T}}(B-\mathcal{T} A)}(x)=\left\{\begin{array}{c}
S\left(1-J_{S}\left(\nu_{A}(x), \nu_{B}(x)\right), I_{\top}\left(\mu_{B}(x), \mu_{A}(x)\right)\right), \\
\quad \text { if } \mu_{A}(x) \leq \mu_{B}(x) \text { and } \nu_{A}(x) \leq \nu_{B}(x), \\
S\left(I_{\top}\left(\mu_{A}(x), \mu_{B}(x)\right), 1-J_{S}\left(\nu_{B}(x), \nu_{A}(x)\right)\right), \\
\text { if } \mu_{A}(x) \geq \mu_{B}(x) \text { and } \nu_{A}(x) \geq \nu_{B}(x), \\
1, \quad \text { otherwise. }
\end{array}\right.
$$

2. If $\mathcal{T}$ is a Lukasiewicz IF-t-norm, then

$$
(A-\mathcal{T} B) \cap_{\mathcal{T}}(B-\mathcal{T} A)=\emptyset
$$

Proof. 1. Recall that for any two intuitionistic fuzzy sets $A$ and $B$, we define the intersection by means of any t-representable IF-t-norm $\mathcal{T}=(\top, S)$ as follows:

$$
A \cap_{\mathcal{T}} B=\left\{\left\langle x, \top\left(\mu_{A}(x), \mu_{B}(x)\right), S\left(\nu_{A}(x), \nu_{B}(x)\right)\right\rangle \mid x \in X\right\} .
$$

So,

$$
\begin{array}{r}
\mu_{(A-\mathcal{T} B) \cap_{\mathcal{T}}(B-\mathcal{T} A)}(x)=\top\left(J_{S}\left(\nu_{A}(x), \nu_{B}(x)\right), J_{S}\left(\nu_{B}(x), \nu_{A}(x)\right)\right), \\
\nu_{(A-\mathcal{T} B) \cap_{\mathcal{T}}(B-\mathcal{T} A)}(x)=S\left(\min \left\{I_{\top}\left(\mu_{A}(x), \mu_{B}(x)\right), 1-J_{S}\left(\nu_{A}(x), \nu_{B}(x)\right)\right\},\right. \\
\left.\min \left\{I_{\top}\left(\mu_{B}(x), \mu_{A}(x)\right), 1-J_{S}\left(\nu_{B}(x), \nu_{A}(x)\right)\right\}\right) .
\end{array}
$$

We note that $J_{S}\left(\nu_{A}(x), \nu_{B}(x)\right)=0$, whenever $\nu_{A}(x) \geq \nu_{B}(x)$ and $I_{\top}\left(\mu_{A}(x), \mu_{B}(x)\right)=1$, whenever $\mu_{A}(x) \leq \mu_{B}(x)$, then consider the following cases: for all $x \in X$,

Case i: If $\mu_{A}(x) \leq \mu_{B}(x)$ and $\nu_{A}(x) \leq \nu_{B}(x)$, then from Equation (10) $\mu_{(A-\mathcal{T} B) \cap_{\mathcal{T}}(B-\mathcal{T} A)}(x)=$ $\top\left(J_{S}\left(\nu_{A}(x), \nu_{B}(x)\right), 0\right)=0$, and from Equation (11) we have

$$
\begin{gathered}
\nu_{(A-\mathcal{T} B) \cap_{\mathcal{T}}(B-\mathcal{T} A)}(x)=S\left(\min \left\{1,1-J_{S}\left(\nu_{A}(x), \nu_{B}(x)\right)\right\}, \min \left\{I_{\top}\left(\mu_{B}(x), \mu_{A}(x)\right), 1-0\right\}\right), \\
=S\left(1-J_{S}\left(\nu_{A}(x), \nu_{B}(x)\right), I_{\top}\left(\mu_{B}(x), \mu_{A}(x)\right)\right) .
\end{gathered}
$$


Case ii: If $\mu_{A}(x) \geq \mu_{B}(x)$ and $\nu_{A}(x) \geq \nu_{B}(x)$, then from Equation $(10) \mu_{(A-\mathcal{T} B) \cap_{\mathcal{T}}(B-\mathcal{T} A)}(x)=$ $\top\left(0, J_{S}\left(\nu_{B}(x), \nu_{A}(x)\right)\right)=0$, and from Equation (11)

$$
\begin{gathered}
\nu_{(A-\mathcal{T} B) \cap_{\mathcal{T}}(B-\mathcal{T} A)}(x)=S\left(\min \left\{I_{\top}\left(\mu_{A}(x), \mu_{B}(x)\right), 1-0\right\}, \min \left\{1,1-J_{S}\left(\nu_{B}(x), \nu_{A}(x)\right)\right\}\right), \\
=S\left(I_{\top}\left(\mu_{A}(x), \mu_{B}(x)\right), 1-J_{S}\left(\nu_{B}(x), \nu_{A}(x)\right)\right) .
\end{gathered}
$$

Other possible cases are:

Case iii: If $\mu_{A}(x) \leq \mu_{B}(x)$ and $\nu_{A}(x) \geq \nu_{B}(x)$, then from Equation $(10) \mu_{(A-\mathcal{T} B) \cap_{\mathcal{T}}(B-\mathcal{T} A)}(x)=$ $\top\left(0, J_{S}\left(\nu_{B}(x), \nu_{A}(x)\right)\right)=0$, and from Equation (11)

$$
\begin{gathered}
\nu_{(A-\mathcal{T} B) \cap_{\mathcal{T}}(B-\mathcal{T} A)}(x)=S\left(\min \{1,1-0\}, \min \left\{I_{\top}\left(\mu_{B}(x), \mu_{A}(x)\right), 1-J_{S}\left(\nu_{B}(x), \nu_{A}(x)\right)\right\}\right), \\
=S\left(1, \min \left\{I_{\top}\left(\mu_{B}(x), \mu_{A}(x)\right), 1-J_{S}\left(\nu_{B}(x), \nu_{A}(x)\right)\right\}\right)=1 .
\end{gathered}
$$

Case iv: If $\mu_{A}(x) \geq \mu_{B}(x)$ and $\nu_{A}(x) \leq \nu_{B}(x)$, then from Equation (10) $\mu_{(A-\mathcal{\tau} B) \cap_{\mathcal{T}}(B-\mathcal{T} A)}(x)=$ $\top\left(J_{S}\left(\nu_{A}(x), \nu_{B}(x)\right), 0\right)=0$, and from Equation (11)

$$
\begin{gathered}
\nu_{(A-\mathcal{T} B) \cap_{\mathcal{T}}(B-\mathcal{T} A)}(x)=S\left(\min \left\{I_{\top}\left(\mu_{A}(x), \mu_{B}(x)\right), 1-J_{S}\left(\nu_{A}(x), \nu_{B}(x)\right)\right\}, \min \{1,1-0\}\right), \\
=S\left(\min \left\{I_{\top}\left(\mu_{A}(x), \mu_{B}(x)\right), 1-J_{S}\left(\nu_{A}(x), \nu_{B}(x)\right)\right\}, 1\right)=1 .
\end{gathered}
$$

So, we have established the result 1.

2. If $\mathcal{T}$ is Lukasiewicz IF-t-norm, then $\mathcal{T}=\mathcal{T}_{L}=\left(\top_{L}, S_{L}\right)$. Since from the result in 1 above, we have the membership function $\mu_{(A-\mathcal{T} B) \cap_{\mathcal{T}}(B-\mathcal{T} A)}(x)=0, \forall x \in X$, then from Equation (9) it suffices to prove that the non-membership function, $\nu_{(A-\mathcal{T} B) \cap \mathcal{T}(B-\mathcal{T} A)}(x)=1, \forall x \in$ $X$, for the first two cases in (9). From Equation (11),

i If $\mu_{A}(x) \leq \mu_{B}(x)$ and $\nu_{A}(x) \leq \nu_{B}(x)$, we obtain by applying Proposition 9 and Example 8,

$$
\begin{gathered}
\nu_{(A-\mathcal{T} B) \cap_{\mathcal{T}}(B-\mathcal{T} A)}(x)=\min \left(1-J_{S_{L}}\left(\nu_{A}(x), \nu_{B}(x)\right)+I_{\top_{L}}\left(\mu_{B}(x), \mu_{A}(x)\right), 1\right), \\
=1, \quad \text { if } \mu_{A}(x)=\mu_{B}(x) \text { or } \nu_{A}(x)=\nu_{B}(x) .
\end{gathered}
$$

If $\mu_{A}(x)<\mu_{B}(x)$ and $\nu_{A}(x)<\nu_{B}(x)$, then we have

$$
\begin{gathered}
\nu_{(A-\mathcal{T} B) \cap_{\mathcal{T}}(B-\mathcal{T} A)}(x)=\min \left(1-\nu_{B}(x)+\nu_{A}(x)+1-\mu_{B}(x)+\mu_{A}(x), 1\right), \\
=\min \left(2-\left(\mu_{B}(x)+\nu_{B}(x)\right)+\mu_{A}(x)+\nu_{A}(x), 1\right)=1, \quad \text { since } \mu_{B}(x)+\nu_{B}(x) \leq 1 .
\end{gathered}
$$

ii If $\mu_{A}(x) \geq \mu_{B}(x)$ and $\nu_{A}(x) \geq \nu_{B}(x)$, we obtain by applying Proposition 9 and Example 8,

$$
\begin{gathered}
\nu_{(A-\mathcal{T} B) \cap_{\mathcal{T}}(B-\mathcal{T} A)}(x)=\min \left(I_{\top_{L}}\left(\mu_{A}(x), \mu_{B}(x)\right)+1-J_{S_{L}}\left(\nu_{B}(x), \nu_{A}(x)\right), 1\right), \\
=1, \quad \text { if } \mu_{A}(x)=\mu_{B}(x) \text { or } \nu_{A}(x)=\nu_{B}(x) .
\end{gathered}
$$

If $\mu_{A}(x)>\mu_{B}(x)$ and $\nu_{A}(x)>\nu_{B}(x)$, then we have

$$
\begin{gathered}
\nu_{(A-\mathcal{T} B) \cap_{\mathcal{T}}(B-\mathcal{T} A)}(x)=\min \left(1-\mu_{A}(x)+\mu_{B}(x)+1-\nu_{A}(x)+\nu_{B}(x), 1\right), \\
=\min \left(2-\left(\mu_{A}(x)+\nu_{A}(x)\right)+\mu_{B}(x)+\nu_{B}(x), 1\right)=1, \quad \text { since } \mu_{A}(x)+\nu_{A}(x) \leq 1 .
\end{gathered}
$$


So $\left(\mu_{(A-\mathcal{T} B) \cap_{\mathcal{T}}(B-\mathcal{T} A)}(x), \nu_{(A-\mathcal{T} B) \cap_{\mathcal{T}}(B-\mathcal{T} A)}(x)\right)=(0,1)$, for all $x \in X$. Hence result 2 is established.

Remark 1. 1. Note that, $\left(A-{ }_{\mathcal{T}} B\right) \cap_{\mathcal{T}}(B-\mathcal{T} A)=\emptyset$ whenever either $A \subseteq B$ or $B \subseteq A$. This follows immediately from the third case in Equation (9).

2. Proposition 2 specifies conditions which are preserved by the intuitionistic fuzzy difference operation. These four conditions shall be referred to as the minimal conditions to require of difference operation on (even in crisp, fuzzy and intuitionistic) sets in general.

The following result gives a necessary and sufficient condition for difference of intuitionistic fuzzy sets to be a fuzzy set.

Proposition 4. Let $A$ and $B$ be any intuitionistic fuzzy sets defined on $X$. Then the intuitionistic fuzzy difference $A-\mathcal{T} B$ is a fuzzy set if and only if for all $x \in X$,

$$
I_{\top}\left(\mu_{A}(x), \mu_{B}(x)\right) \geq 1-J_{S}\left(\nu_{A}(x), \nu_{B}(x)\right) .
$$

Proof. Let $x \in X$. Then from the Definition 8,

$\left(\mu_{A-\mathcal{T} B}(x), \nu_{A-\mathcal{T} B}(x)\right)=\left(J_{S}\left(\nu_{A}(x), \nu_{B}(x)\right), \min \left\{I_{\top}\left(\mu_{A}(x), \mu_{B}(x)\right), 1-J_{S}\left(\nu_{A}(x), \nu_{B}(x)\right)\right\}\right)$.

$A-\mathcal{T} B$ is a fuzzy set if and only if $\nu_{A-\mathcal{T} B}(x)=1-\mu_{A-\mathcal{T} B}(x)$,

if and only if

$\min \left\{I_{\top}\left(\mu_{A}(x), \mu_{B}(x)\right), 1-J_{S}\left(\nu_{A}(x), \nu_{B}(x)\right)\right\}=1-J_{S}\left(\nu_{A}(x), \nu_{B}(x)\right)$,

if and only if $I_{\top}\left(\mu_{A}(x), \mu_{B}(x)\right) \geq 1-J_{S}\left(\nu_{A}(x), \nu_{B}(x)\right)$.

Note that $A-\mathcal{T} B$ also becomes a fuzzy set if $A \subset B$, because in this case $A-\mathcal{\tau} B=\emptyset$ (Proposition 2), $I_{\top}\left(\mu_{A}(x), \mu_{B}(x)=1\right.$ and $J_{S}\left(\nu_{A}(x), \nu_{B}(x)\right)=0$. Furthermore, in the case where $A-\mathcal{T} B$ becomes a fuzzy set, we deduce from Proposition 4 that for

$$
x \in X:\left(\mu_{A-\mathcal{T} B}(x), \nu_{A-\mathcal{T} B}(x)\right)=\left(J_{S}\left(\nu_{A}(x), \nu_{B}(x)\right), 1-J_{S}\left(\nu_{A}(x), \nu_{B}(x)\right)\right) .
$$

This can be considered as fuzzy part of $A-\mathcal{T} B$.

The following are typical applications of Proposition 4 to difference operators associated with the three usual and well-known $\mathcal{T}$.

Example 5. For any intuitionistic fuzzy sets $A$ and $B$ defined on $X$,

1. The difference operation associated with $\mathcal{T}_{M}$ is given by, for all $x \in X$

$$
\left(\mu_{A-\tau_{M} B}(x), \nu_{A-\tau_{M} B}(x)\right)= \begin{cases}\left(\nu_{B}(x), \mu_{B}(x)\right), & \text { Intuitionistic Fuzzy Part } \\ \left(\nu_{B}(x), 1-\nu_{B}(x)\right), & \text { Fuzzy Part } .\end{cases}
$$


2. The difference operation associated with $\mathcal{T}_{P}$ is given by, for all $x \in X$

$$
\left(\mu_{A-\mathcal{T}_{P} B}(x), \nu_{A-\mathcal{T}_{P} B}(x)\right)=\left\{\begin{array}{lc}
\left(\frac{\nu_{B}(x)-\nu_{A}(x)}{1-\nu_{A}(x)}, \frac{\mu_{B}(x)}{\mu_{A}(x)}\right), & \text { Intuitionistic Fuzzy Part } \\
\left.\frac{\nu_{B}(x)-\nu_{A}(x)}{1-\nu_{A}(x)}, \frac{1-\nu_{B}(x)}{1-\nu_{A}(x)}\right), & \text { Fuzzy Part. }
\end{array}\right.
$$

3. The difference operation associated with $\mathcal{T}_{L}$ is given by, for all $x \in X$

$$
\left(\mu_{A-\mathcal{\tau}_{L} B}(x), \nu_{A-\mathcal{T}_{L} B}(x)\right)=\left\{\begin{array}{l}
\left(\nu_{B}(x)-\nu_{A}(x), 1-\mu_{A}(x)+\mu_{B}(x)\right), \text { Intuitionistic Fuzzy } \\
\left(\nu_{B}(x)-\nu_{A}(x), 1+\nu_{A}(x)-\nu_{B}(x)\right), \text { Fuzzy Part. }
\end{array}\right.
$$

Notation 1. Let $A$ and $B$ be any fuzzy sets, $A \equiv B$ if and only if for all $x \in X, \mu_{A}(x)=\mu_{B}(x)$.

The following result shows that the intuitionistic fuzzy difference operator defined in Definition 8 associated with t-representable IF t-norm $\mathcal{T}=(\top, S)$ is a generalization of fuzzy difference operator proposed by Fono et al. [10] associated with a t-norm $T$ if and only if the fuzzy t-norm T and fuzzy t-conorm $S$ are dual.

Proposition 5 (Generalization of Difference Operation for Fuzzy Sets). Let $\top$ and $S$ be any $t$ norm and t-conorm respectively, and $\mathcal{T}=(T, S)$ be a t-representable IF t-norm associated with any intuitionistic fuzzy set. $T$ and $S$ are dual if and only if for any fuzzy sets $A$ and $B, A-\mathcal{T} B$ is a fuzzy set and $A-\top B \equiv A-\mathcal{T} B$.

Proof. Let $x \in X$, and $A$ and $B$ be any fuzzy sets.

a. Assume that $\top$ and $S$ are dual.

i. Let us show that $A-\mathcal{T} B$ is a fuzzy set.

Since $\top$ and $S$ are dual, then From Proposition 10, $I_{\top}\left(\mu_{A}(x), \mu_{B}(x)\right)=1-J_{S}(1-$ $\left.\mu_{A}(x), 1-\mu_{B}(x)\right)$ and from Proposition 4 , the result follows.

ii. Now we shall show that, $A-_{\top} B \equiv A-_{\mathcal{T}} B$. It is sufficient to prove that $\mu_{A-_{\top} B}(x)=$ $\mu_{A-\mathcal{T} B}(x)$.

According to Fono and al. [10], $\mu_{A-\top B}(x)=1-I_{\top}\left(\mu_{A}(x), \mu_{B}(x)\right)$ and from Definition $8 \mu_{A-\mathcal{T} B}(x)=J_{S}\left(1-\mu_{A}(x), 1-\mu_{B}(x)\right)$.

Since $\top$ and $S$ are dual, the Proposition 10 shows that, $I_{\top}\left(\mu_{A}(x), \mu_{B}(x)\right)=1-J_{S}(1-$ $\left.\mu_{A}(x), 1-\mu_{B}(x)\right)$ and the result follows.

b. Assume now that $A-_{\mathcal{T}} B$ is a fuzzy set and $A-_{\top} B \equiv A-_{\mathcal{T}} B$. Let us show that $\top$ and $S$ are dual.

We have,

$$
\begin{aligned}
\mu_{A-\top B}(x) & =1-I_{\top}\left(\mu_{A}(x), \mu_{B}(x)\right) \\
& =1-\max \left\{t \in[0 ; 1], \top\left(\mu_{A}(x), t\right) \leq \mu_{B}(x)\right\} \\
& =\min \left\{1-t \in[0 ; 1], \top\left(\mu_{A}(x), t\right) \leq \mu_{B}(x)\right\} \\
& =\min \left\{1-t \in[0 ; 1], \top\left(\mu_{A}(x), t\right) \leq \mu_{B}(x)\right\},
\end{aligned}
$$


and

$$
\begin{aligned}
\mu_{A-\mathcal{T} B}(x) & =J_{S}\left(1-\mu_{A}(x), 1-\mu_{B}(x)\right) \\
& =\min \left\{r \in[0 ; 1], S\left(1-\mu_{A}(x), r\right) \geq 1-\mu_{B}(x)\right\} \\
& =\min \left\{1-t \in[0 ; 1], S\left(1-\mu_{A}(x), 1-t\right) \geq 1-\mu_{B}(x)\right\} \\
& =\min \left\{1-t \in[0 ; 1], 1-S\left(1-\mu_{A}(x), 1-t\right) \leq \mu_{B}(x)\right\}
\end{aligned}
$$

Since $A-_{\top} B \equiv A-_{\mathcal{T}} B$ then, $\mu_{A-\top B}(x)=\mu_{A-\mathcal{T} B}(x)$. From Equations (12) and (13) $\top\left(\mu_{A}(x), t\right)=1-S\left(1-\mu_{A}(x), 1-t\right), \forall t \in[0 ; 1]$ and the result follows.

In the following, we define a new symmetric difference operation for intuitionistic fuzzy sets based on the IF-R-implication and IF-co-implication and we study its properties.

\subsection{Definitions and properties of symmetric difference operations}

The idea for the new definition is derived from the classical formula for symmetric difference and the operations of union and intersection alongside with the proposed difference for intuitionistic fuzzy sets in Section 3.

Definition 9. Let $A, B$ be any two intuitionistic fuzzy sets defined on $X$. The intuitionistic fuzzy symmetric difference associated to $\mathcal{T}$ of $A$ and $B$ is the intuitionistic fuzzy set on $X$ denoted by $A \triangle \mathcal{T} B$ and defined by the membership and non-membership degrees as follows:

For all $x \in X$,

$$
\begin{array}{r}
\mu_{A \triangle \mathcal{T} B}(x)=J_{S}\left(\nu_{A}(x) \wedge \nu_{B}(x), \nu_{A}(x) \vee \nu_{B}(x)\right), \\
\nu_{A \triangle \mathcal{T} B}(x)=\min \left\{I_{\top}\left(\mu_{A}(x) \vee \mu_{B}(x), \mu_{A}(x) \wedge \mu_{B}(x)\right),\right. \\
\left.1-J_{S}\left(\nu_{A}(x) \wedge \nu_{B}(x), \nu_{A}(x) \vee \nu_{B}(x)\right)\right\} .
\end{array}
$$

In what follows, we establish some results showing that some properties of the classical set symmetric difference are preserved by this new proposed intuitionistic fuzzy symmetric difference operation.

Proposition 6 (Properties of Intuitionistic Fuzzy Symmetric Difference Operation). Let A, B be any intuitionistic fuzzy sets on $X$. The following properties for intuitionistic fuzzy symmetric difference operation hold:

1. $A \triangle \mathcal{T} B=(A-\mathcal{T} B) \cup(B-\mathcal{T} A)$;

2. $A \triangle_{\mathcal{T}} B=B \triangle_{\mathcal{T}} A$;

3. If $A \subseteq B$, then $A \triangle_{\mathcal{T}} B=B-\mathcal{T} A$;

4. $A \triangle \mathcal{T} A=\emptyset$. 
Proof. 1. The following are properties for fuzzy-R-implication, $I_{\top}$ and fuzzy co-implication, $J_{S}$ which we require here:

$$
\begin{aligned}
& I_{\top}(a \vee b, c)=I_{\top}(a, c) \wedge I_{\top}(b, c), \text { and } J_{S}(a \vee b, c)=J_{S}(a, c) \wedge J_{S}(b, c) ; \\
& I_{\top}(a \wedge b, c)=I_{\top}(a, c) \vee I_{\top}(b, c), \text { and } J_{S}(a \wedge b, c)=J_{S}(a, c) \vee J_{S}(b, c) ; \\
& I_{\top}(a, b \vee c)=I_{\top}(a, b) \vee I_{\top}(a, c), \text { and } J_{S}(a, b \vee c)=J_{S}(a, b) \vee J_{S}(a, c) ; \\
& I_{\top}(a, b \wedge c)=I_{\top}(a, b) \wedge I_{\top}(a, c), \text { and } J_{S}(a, b \wedge c)=J_{S}(a, b) \wedge J_{S}(a, c) .
\end{aligned}
$$

These can easily be verified.

Now, we proceed to prove 1 and 2 consequently as follows: From Equation (14) and applying above properties of $I_{\top}$ and $J_{S}$ we have, for all $x \in X$

$$
\begin{gathered}
\left(\mu_{A \triangle_{\mathcal{T}} B}(x), \nu_{A \triangle_{\mathcal{T}} B}(x)\right)=\left(J_{S}\left(\nu_{A}(x) \wedge \nu_{B}(x), \nu_{A}(x)\right) \vee J_{S}\left(\nu_{A}(x) \wedge \nu_{B}(x), \nu_{B}(x)\right),\right. \\
\min \left\{I_{\top}\left(\mu_{A}(x) \vee \mu_{B}(x), \mu_{A}(x)\right) \wedge I_{\top}\left(\mu_{A}(x) \vee \mu_{B}(x), \mu_{B}(x)\right)\right. \\
\left.\left.1-J_{S}\left(\nu_{A}(x) \wedge \nu_{B}(x), \nu_{A}(x)\right) \vee J_{S}\left(\nu_{A}(x) \wedge \nu_{B}(x), \nu_{B}(x)\right)\right\}\right) .
\end{gathered}
$$

So we have

$$
\begin{gathered}
\mu_{A \triangle \mathcal{T} B}(x)=\left(J_{S}\left(\nu_{A}(x), \nu_{A}(x)\right) \vee J_{S}\left(\nu_{B}(x), \nu_{A}(x)\right)\right) \vee\left(J_{S}\left(\nu_{A}(x), \nu_{B}(x)\right) \vee J_{S}\left(\nu_{B}(x), \nu_{B}(x)\right)\right), \\
\nu_{A \triangle_{\mathcal{T}} B}(x)=\min \left\{( I _ { \top } ( \mu _ { A } ( x ) , \mu _ { A } ( x ) ) \wedge I _ { \top } ( \mu _ { B } ( x ) , \mu _ { A } ( x ) ) \} \wedge \left\{I_{\top}\left(\mu_{A}(x), \mu_{B}(x)\right)\right.\right. \\
\left.\wedge I_{\top}\left(\mu_{B}(x), \mu_{B}(x)\right)\right), 1-\left(J_{S}\left(\nu_{A}(x), \nu_{A}(x)\right) \vee J_{S}\left(\nu_{B}(x), \nu_{A}(x)\right)\right\} \\
\vee \\
\left.\vee\left\{J_{S}\left(\nu_{A}(x), \nu_{B}(x)\right) \vee J_{S}\left(\nu_{B}(x), \nu_{B}(x)\right)\right)\right\}
\end{gathered}
$$

and applying Proposition 9 we have the following:

$$
\begin{aligned}
& \mu_{A \triangle_{\mathcal{T} B}}(x)=J_{S}\left(\nu_{B}(x), \nu_{A}(x)\right) \vee J_{S}\left(\nu_{A}(x), \nu_{B}(x)\right), \\
& =J_{S}\left(\nu_{A}(x), \nu_{B}(x)\right) \vee J_{S}\left(\nu_{B}(x), \nu_{A}(x)\right) \text {, } \\
& =\mu_{A-\mathcal{T} B}(x) \vee \mu_{B-\mathcal{T} A}(x) \text {, } \\
& =\mu_{(A-\mathcal{T} B) \cup(B-\mathcal{T} A)}(x) \text {. }
\end{aligned}
$$

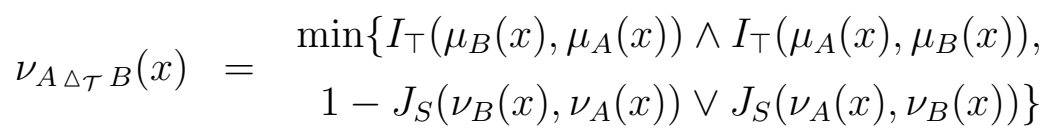

$$
\begin{aligned}
& \begin{array}{r}
=\min \left\{I_{\top}\left(\mu_{A}(x), \mu_{B}(x)\right) \wedge I_{\top}\left(\mu_{B}(x), \mu_{A}(x)\right),\right. \\
\left.1-J_{S}\left(\nu_{A}(x), \nu_{B}(x)\right) \vee J_{S}\left(\nu_{B}(x), \nu_{A}(x)\right)\right\}
\end{array} \\
& =\min \left\{I_{\top}\left(\mu_{A}(x), \mu_{B}(x)\right) \wedge\left(1-J_{S}\left(\nu_{A}(x), \nu_{B}(x)\right)\right)\right. \text {, } \\
& \left.I_{\top}\left(\mu_{B}(x), \mu_{A}(x)\right) \wedge\left(1-J_{S}\left(\nu_{B}(x), \nu_{A}(x)\right)\right)\right\} \\
& =\nu_{A-\mathcal{T} B}(x) \wedge \nu_{B-\mathcal{T} A}(x) \\
& =\nu_{(A-\mathcal{T} B) \cup(B-\mathcal{T} A)}(x) \text {. }
\end{aligned}
$$

So, result 1 is established. 
2. By commutativity of Equations (15) and (16), result 2 follows, since $A \Delta \mathcal{T} B=(A-\mathcal{T} B) \cup(B-\mathcal{T} A)=(B-\mathcal{T} A) \cup(A-\mathcal{T} B)=B \Delta \mathcal{T} A$.

3. If $A \subseteq B$, then for all $x \in X, \mu_{A}(x) \leq \mu_{B}(x)$ and $\nu_{A}(x) \geq \nu_{B}(x)$. Applying the above inequalities to the Equation (14), we get

$$
\begin{aligned}
\left(\mu_{A \triangle_{\mathcal{T}} B}(x), \nu_{A \triangle_{\mathcal{T}} B}(x)\right) & =\left(J_{S}\left(\nu_{B}(x), \nu_{A}(x)\right), \min \left\{I_{\top}\left(\mu_{B}(x), \mu_{A}(x)\right), 1-J_{S}\left(\nu_{B}(x), \nu_{A}(x)\right)\right\}\right) \\
& =\left(\mu_{B-\mathcal{T} A}(x), \nu_{B-\mathcal{T} A}(x)\right)
\end{aligned}
$$

and the result follows.

4. By Equation (14) we have, for all $x \in X$

$$
\begin{gathered}
\mu_{A \triangle \mathcal{T} A}(x)=J_{S}\left(\nu_{A}(x) \wedge \nu_{A}(x), \nu_{A}(x) \vee \nu_{A}(x)\right) \\
=J_{S}\left(\nu_{A}(x), \nu_{A}(x)\right)=0 . \\
\nu_{A \Delta \mathcal{T} A}(x)=\min \left\{I_{\top}\left(\mu_{A}(x) \vee \mu_{A}(x), \mu_{A}(x) \wedge \mu_{A}(x)\right), 1-J_{S}\left(\nu_{A}(x) \wedge \nu_{A}(x), \nu_{A}(x) \vee \nu_{A}(x)\right)\right\} \\
=\min \left\{I_{\top}\left(\mu_{A}(x), \mu_{A}(x)\right), 1-J_{S}\left(\nu_{A}(x), \nu_{A}(x)\right)\right\} \\
=\min \{1,1\}=1 .
\end{gathered}
$$

So the result is established.

The following are typical examples of symmetric difference operators associated with the three usual and well-known $\mathcal{T}$.

Example 6. For any two intuitionistic fuzzy sets $A$ and $B$ defined on $X$,

1. The symmetric difference operator associated with $\mathcal{T}_{M}$ is given by, for all $x \in X$

$$
\begin{gathered}
\mu_{A \triangle \mathcal{T}_{M} B}(x)=\left\{\begin{array}{l}
0, \quad \text { if }\left(\mu_{A}(x), \nu_{A}(x)\right)=\left(\mu_{B}(x), \nu_{B}(x)\right) \\
\nu_{B}(x) \vee \nu_{A}(x), \quad \text { if }\left(\mu_{A}(x), \nu_{A}(x)\right) \neq\left(\mu_{B}(x), \nu_{B}(x)\right),
\end{array}\right. \\
\nu_{A \triangle \mathcal{T}_{M} B}(x)=\left\{\begin{array}{c}
1, \quad \text { if }\left(\mu_{A}(x), \nu_{A}(x)\right)=\left(\mu_{B}(x), \nu_{B}(x)\right) \\
\min \left\{\min \left\{\mu_{B}(x), 1-\nu_{B}(x)\right\}, \min \left\{\mu_{A}(x), 1-\nu_{A}(x)\right\}\right\}, \\
\text { if }\left(\mu_{A}(x), \nu_{A}(x)\right) \neq\left(\mu_{B}(x), \nu_{B}(x)\right),
\end{array}\right. \\
=\left\{\begin{array}{c}
1, \quad \text { if }\left(\mu_{A}(x), \nu_{A}(x)\right)=\left(\mu_{B}(x), \nu_{B}(x)\right) \\
\min \left\{\mu_{A}(x) \wedge \mu_{B}(x), 1-\nu_{A}(x) \vee \nu_{B}(x)\right\}, \quad \text { if }\left(\mu_{A}(x), \nu_{A}(x)\right) \neq\left(\mu_{B}(x), \nu_{B}(x)\right) .
\end{array}\right.
\end{gathered}
$$

2. The symmetric difference operator associated with $\mathcal{T}_{P}$ is given by, for all $x \in X$

$$
\mu_{A \triangle \mathcal{T}_{P} B}(x)=\left\{\begin{array}{l}
0, \quad \text { if }\left(\mu_{A}(x), \nu_{A}(x)\right)=\left(\mu_{B}(x), \nu_{B}(x)\right) \\
\max \left\{\frac{\nu_{B}(x)-\nu_{A}(x)}{1-\nu_{A}(x)}, \frac{\nu_{A}(x)-\nu_{B}(x)}{1-\nu_{B}(x)}\right\}, \quad \text { if }\left(\mu_{A}(x), \nu_{A}(x)\right) \neq\left(\mu_{B}(x), \nu_{B}(x)\right),
\end{array}\right.
$$




$$
\begin{aligned}
& = \begin{cases}0, \quad \text { if }\left(\mu_{A}(x), \nu_{A}(x)\right)= & \left(\mu_{B}(x), \nu_{B}(x)\right) \\
\frac{\left(\nu_{A}(x)-\nu_{B}(x)\right) \vee\left(\nu_{B}(x)-\nu_{A}(x)\right)}{\left(1-\nu_{A}(x)\right) \wedge\left(1-\nu_{B}(x)\right)}, & \text { if }\left(\mu_{A}(x), \nu_{A}(x)\right) \neq\left(\mu_{B}(x), \nu_{B}(x)\right),\end{cases} \\
& \nu_{A \triangle \mathcal{T}_{P} B}(x)=\left\{\begin{aligned}
1, & \text { if }\left(\mu_{A}(x), \nu_{A}(x)\right)=\left(\mu_{B}(x), \nu_{B}(x)\right) \\
\min \left\{\min \left\{\frac{\mu_{B}(x)}{\mu_{A}(x)}, \frac{\mu_{A}(x)}{\mu_{B}(x)}\right\}, 1-\max \left\{\frac{\nu_{B}(x)-\nu_{A}(x)}{1-\nu_{A}(x)}, \frac{\nu_{A}(x)-\nu_{B}(x)}{1-\nu_{B}(x)}\right\}\right\}, & \\
& \text { if }\left(\mu_{A}(x), \nu_{A}(x)\right) \neq\left(\mu_{B}(x), \nu_{B}(x)\right),
\end{aligned}\right. \\
& =\left\{\begin{array}{c}
1, \quad \text { if }\left(\mu_{A}(x), \nu_{A}(x)\right)=\left(\mu_{B}(x), \nu_{B}(x)\right) \\
\min \left\{\frac{\mu_{A}(x) \wedge \mu_{B}(x)}{\mu_{A}(x) \vee \mu_{B}(x)}, 1-\frac{\left(\nu_{A}(x)-\nu_{B}(x)\right) \vee\left(\nu_{B}(x)-\nu_{A}(x)\right)}{\left(1-\nu_{A}(x)\right) \wedge\left(1-\nu_{B}(x)\right)}\right\} \\
\quad \text { if }\left(\mu_{A}(x), \nu_{A}(x)\right) \neq\left(\mu_{B}(x), \nu_{B}(x)\right) .
\end{array}\right.
\end{aligned}
$$

3. The symmetric difference operator associated with $\mathcal{T}_{L}$ is given by, for all $x \in X$

$$
\begin{aligned}
& \mu_{A \triangle \mathcal{T}_{L} B}(x)=\left\{\begin{array}{l}
0, \quad \text { if }\left(\mu_{A}(x), \nu_{A}(x)\right)=\left(\mu_{B}(x), \nu_{B}(x)\right) \\
\left(\nu_{A}(x)-\nu_{B}(x)\right) \vee\left(\nu_{B}(x)-\nu_{A}(x)\right), \quad \text { if }\left(\mu_{A}(x), \nu_{A}(x)\right) \neq\left(\mu_{B}(x), \nu_{B}(x)\right),
\end{array}\right. \\
& \nu_{A \triangle_{\mathcal{T}_{L}} B}(x)=\left\{\begin{array}{l}
1, \quad \text { if }\left(\mu_{A}(x), \nu_{A}(x)\right)=\left(\mu_{B}(x), \nu_{B}(x)\right) \\
\min \left\{\min \left\{1-\mu_{A}(x)+\mu_{B}(x), 1+\nu_{A}(x)-\nu_{B}(x)\right\},\right. \\
\left.\min \left\{1-\mu_{B}(x)+\mu_{A}(x), 1+\nu_{B}(x)-\nu_{A}(x)\right\}\right\} \\
\quad \text { if }\left(\mu_{A}(x), \nu_{A}(x)\right) \neq\left(\mu_{B}(x), \nu_{B}(x)\right),
\end{array}\right. \\
& =\left\{\begin{array}{l}
1, \quad \text { if }\left(\mu_{A}(x), \nu_{A}(x)\right)=\left(\mu_{B}(x), \nu_{B}(x)\right) \\
\min \left\{1-\left(\mu_{A}(x)-\mu_{B}(x)\right) \vee\left(\mu_{B}(x)-\mu_{A}(x)\right),\right. \\
\left.1-\left(\nu_{A}(x)-\nu_{B}(x)\right) \vee\left(\nu_{B}(x)-\nu_{A}(x)\right)\right\}, \text { if }\left(\mu_{A}(x), \nu_{A}(x)\right) \neq\left(\mu_{B}(x), \nu_{B}(x)\right) .
\end{array}\right.
\end{aligned}
$$

The following result shows that, the intuitionistic fuzzy symmetric difference operator in Definition 9 associated with t-representable IF t-norm $\mathcal{T}=(T, S)$ is a generalization of fuzzy symmetric difference operator proposed by Fono et al. [10] associated with a t-norm $\top$ if and only if the fuzzy t-norm $\top$ and fuzzy t-conorm $S$ are dual.

Proposition 7 (Generalization of Symmetric Difference Operation for Fuzzy Sets). Let $\top$ and $S$ be any t-norm and t-conorm, respectively, and $\mathcal{T}=(T, S)$ be a t-representable IF t-norm associated with any intuitionistic fuzzy set. $T$ and $S$ are dual if and only if for any fuzzy sets $C$ and $D, C \triangle_{\mathcal{T}} D$ is a fuzzy set and $C \triangle_{\top} D \equiv C \triangle_{\mathcal{T}} D$.

Proof. Let $x \in X$, and $C$ and $D$ be any fuzzy sets.

a. Assume that $\top$ and $S$ are dual.

i. Let us show that $C \triangle \mathcal{T} D$ is a fuzzy set.

Since $C$ and $D$ are fuzzy sets $\left(1-\mu_{C}(x)=\nu_{C}(x)\right.$ and $\left.1-\mu_{D}(x)=\nu_{D}(x)\right)$, and $\top$ and 
$S$ are dual, then from Proposition 10,

$$
\begin{array}{r}
I_{\top}\left(\mu_{C}(x) \vee \mu_{D}(x), \mu_{C}(x) \wedge \mu_{D}(x)\right)=1-J_{S}\left(1-\mu_{C}(x) \vee \mu_{D}(x), 1-\mu_{C}(x) \wedge \mu_{D}(x)\right) \\
=1-J_{S}\left(\left(1-\mu_{C}(x)\right) \wedge\left(1-\mu_{D}(x)\right),\left(1-\mu_{C}(x)\right) \vee\left(1-\mu_{D}(x)\right)\right) \\
=1-J_{S}\left(\nu_{C}(x) \wedge \nu_{D}(x), \nu_{C}(x) \vee \nu_{D}(x)\right) .
\end{array}
$$

From Definition 9, the result follows.

ii. Now we shall show that, $C \triangle_{\top} D \equiv C \triangle_{\mathcal{T}} D$. It is sufficient to prove that $\mu_{C \triangle_{\top} D}(x)=$ $\mu_{C \Delta_{\mathcal{T}} D}(x)$.

Definition 7 and Definition 9 show that,

$$
\mu_{C \underset{\top}{\Delta} D}(x)=\mu_{C \cup D \underset{\top}{-} C \cap D}(x)= \begin{cases}1-I_{\top}^{1}\left(\mu_{C}(x) \vee \mu_{D}(x), \mu_{C}(x) \wedge \mu_{D}(x)\right), & \text { if } i=1 \\ 1-I_{\top}^{2}\left(\mu_{C}(x) \vee \mu_{D}(x), \mu_{C}(x) \wedge \mu_{D}(x)\right), & \text { if } i=2,\end{cases}
$$

and $\mu_{C \triangle_{\mathcal{T}} D}(x)=J_{S}\left(\nu_{C}(x) \wedge \nu_{D}(x), \nu_{C}(x) \vee \nu_{D}(x)\right)$.

From Equation (17), the result follows.

b. Assume now that $C \triangle_{\mathcal{T}} D$ is a fuzzy set and $C \triangle_{\top} D \equiv C \triangle_{\mathcal{T}} D$.

Let us show that $\top$ and $S$ are dual.

We have,

$$
\begin{aligned}
\mu_{C \Delta_{\top} D}(x) & =1-I_{\top}\left(\mu_{C}(x) \vee \mu_{D}(x), \mu_{C}(x) \wedge \mu_{D}(x)\right) \\
& =1-\max \left\{t \in[0 ; 1], \top\left(\mu_{C}(x) \vee \mu_{D}(x), t\right) \leq \mu_{C}(x) \wedge \mu_{D}(x)\right\} \\
& =\min \left\{1-t \in[0 ; 1], \top\left(\mu_{C}(x) \vee \mu_{D}(x), t\right) \leq \mu_{C}(x) \wedge \mu_{D}(x)\right\} \\
& =\min \left\{1-t \in[0 ; 1], \top\left(\mu_{C}(x) \vee \mu_{D}(x), t\right) \leq \mu_{C}(x) \wedge \mu_{D}(x)\right\},
\end{aligned}
$$

and

$$
\begin{aligned}
& \mu_{C} \Delta_{\mathcal{T} D}(x)=J_{S}\left(\nu_{C}(x) \wedge \nu_{D}(x), \nu_{C}(x) \vee \nu_{D}(x)\right) \\
&=\min \left\{r \in[0 ; 1], S\left(\nu_{C}(x) \wedge \nu_{D}(x), r\right) \geq \nu_{C}(x) \vee \nu_{D}(x)\right\} \\
&=\min \left\{1-t \in[0 ; 1], S\left(1-\mu_{C}(x) \vee \mu_{D}(x), 1-t\right) \geq 1-\mu_{C}(x) \wedge \mu_{D}(x)\right\} \\
&=\min \left\{1-t \in[0 ; 1], 1-S\left(1-\mu_{C}(x) \vee \mu_{D}(x), 1-t\right) \leq \mu_{C}(x) \wedge \mu_{D}(x)\right\}
\end{aligned}
$$

Since $C \Delta_{\top} D \equiv C \Delta_{\mathcal{T}} D$ then, $\mu_{C \Delta_{\top} D}(x)=\mu_{C \Delta_{\mathcal{T}} D}(x)$. From Equation (18) and (19), $\top\left(\mu_{C}(x) \vee \mu_{D}(x), t\right)=1-S\left(1-\mu_{C}(x) \vee \mu_{D}(x), 1-t\right), \forall t \in[0 ; 1]$, and the result follows.

In the following Subsection, we investigate some properties of cardinality for intuitionistic fuzzy set difference and symmetric difference. For that, throughout this Subsection, the universal set $X$ is finite. 


\subsection{Some cardinality properties of difference and symmetric difference for IFSs}

We recall the definition and some results on intuitionistic fuzzy cardinality in what follows.

Definition 10 (Cardinality of Intuitionistic Fuzzy Set [19]). Let A be an intuitionistic fuzzy set on $X$. The cardinality of A denoted by $\Sigma \operatorname{count}(A)$ is given by

$$
\Sigma \operatorname{count}(A)=\operatorname{Card}(A)=\left(\sum_{i=1}^{n} \mu_{A}\left(x_{i}\right), \sum_{i=1}^{n} 1-\nu_{A}\left(x_{i}\right)\right) .
$$

One of the properties of this cardinality operation is given here [See Property of $\Sigma$ count [19] ]: Let $A$ and $B$ be any two intuitionistic fuzzy sets on $X$. Then

$$
\Sigma \operatorname{count}(A \cup B)+\Sigma \operatorname{count}(A \cap B)=\Sigma \operatorname{count}(A)+\Sigma \operatorname{count}(B) .
$$

In what follows, we establish a cardinality property that is satisfied by the intuitionistic fuzzy difference and symmetric difference proposed.

Proposition 8. Let $A, B, C$ be any intuitionistic fuzzy sets on $X$. The following property holds:

$$
\operatorname{Card}\left(A \triangle_{\mathcal{T}} B\right) \leq_{L^{*}} \operatorname{Card}\left(A-_{\mathcal{T}} B\right)+\operatorname{Card}(B-\mathcal{T} A)
$$

Proof. Recall from Proposition 6, we have $A \Delta_{\mathcal{T}} B=(A-\mathcal{T} B) \cup(B-\mathcal{T} A)$ and by Equation (21) we obtain

$$
\operatorname{Card}\left(A \triangle_{\mathcal{T}} B\right)=\operatorname{Card}\left(A-_{\mathcal{T}} B\right)+\operatorname{Card}\left(B-_{\mathcal{T}} A\right)-\operatorname{Card}\left(\left(A-_{\mathcal{T}} B\right) \cap\left(B-_{\mathcal{T}} A\right)\right) .
$$

Since by Proposition 3 we have $(A-\mathcal{T} B) \cap_{\mathcal{T}}(B-\mathcal{T} A) \neq \emptyset$ in general, then we have

$$
\operatorname{Card}((A-\mathcal{T} B) \cap(B-\mathcal{T} A)) \geq_{L^{*}} 0_{L^{*}}
$$

Putting Equation (23) into (22) we obtain the required result.

\section{Conclusion}

In this study, we have proposed new difference and symmetric difference operations for intuitionistic fuzzy sets by means of intuitionistic fuzzy R-implications. We have also constructed some examples of difference and symmetric operations associated to the well-known intuitionistic fuzzy t-norms (minimum $\mathcal{T}_{M}$, product $\mathcal{T}_{P}$ and Łukasiewicz $\mathcal{T}_{L}$ ) and established conditions under which these operations yield the same results for fuzzy cases as obtained by Fono et al. [10].

We further established that the intuitionistic fuzzy difference operation preserves four properties out of five, which we referred to as the four minimal conditions to require of a difference operation on sets in general (even in crisp, fuzzy and intuitionistic fuzzy cases). We investigated and established some sufficient conditions under which the fifth property is satisfied. Meanwhile, we established that the intuitionistic fuzzy symmetric difference operation proposed preserves the 
properties of symmetric difference operations for crisp sets and fuzzy sets. We established out of many, one cardinality property that is satisfied by these operations.

The results of Proposition 3 have shown that the property, $(A-\mathcal{T} B) \cap_{\mathcal{T}}(B-\mathcal{T} A)=\emptyset$ do not hold true in general case for the difference operation for intuitionistic fuzzy sets proposed. The open problem will be to determine all intuitionistic fuzzy-t-norms under which the difference operation, so defined, preserves this property. We have not studied here, other cardinality properties and the cardinality-based measures of comparison for intuitionistic fuzzy sets by means of these new difference and symmetric difference operations for intuitionistic fuzzy sets proposed. This area is opened for further research studies.

\section{Acknowledgements}

The authors appreciate the anonymous referees for their assessment of this paper. The First author thanks AIMS Ghana. This paper was initiated when he was an MSc scholar at the Institute. He further appreciate Lord Kavi, who was an advising tutor at the time, for his immense contribution to the success of this research. This work was finalized under the Research Grant No 17-497RG/MATHS/AF/AC-G-FR3240297728 offered by TWAS to the Laboratory of Mathematics of the University of Douala. Prof Fono, Dr Fotso and Mr Dzati sincerely thank TWAS.

\section{References}

[1] Atanassov, K. T. (1986). Intuitionistic fuzzy sets, Fuzzy sets and Systems, 20 (1), 87-96.

[2] Atanassov, K. T. (1999). Intuitionistic fuzzy sets: theory and applications, Physica-Verlag, New York.

[3] Atanassov, K. T. (2010). On intuitionistic fuzzy negations and law for excluded middle, Proceedings of 5th IEEE International Conference Intelligent Systems (IS), 7-9 July 2010, London, United Kingdom, 266-269.

[4] Bustince, H., Kacprzyk, J., \& Mohedano, V. (2000). Intuitionistic fuzzy generators application to intuitionistic fuzzy complementation, Fuzzy sets and systems, 114 (3), 485-504.

[5] Cornelis, C., Deschrijver, G. \& Kerre, E. E. (2004). Implication in intuitionistic fuzzy and interval-valued fuzzy set theory: construction, classification, application, International Journal of Approximate Reasoning, 3 (1), 55-95.

[6] Deschrijver, G., Cornelis, C. \& Kerre, E. E. (2004). On the Representation of Intuitionistic Fuzzy t-Norms and t-Conorms, IEEE Transactions on Fuzzy Systems, 12 (1), 45-61.

[7] Despi, I., Opris, D. \& Yalcin, E. (2013). Generalised Atanassov Intuitionistic Fuzzy Sets, Proceedings of 5th International Conference on Information, Process and Knowledge Management, 24 Feb. - 1 Mar. 2013, Nice, France, 51-52. 
[8] Dubois, D., Gottwald, S., Hajek, P., Kacprzyk, J. \& Prade, H. (2005). Terminological difficulties in fuzzy set theory-the case of intuitionistic fuzzy sets, Fuzzy Sets and Systems, 156 (3), 485-491.

[9] Ejegwa, P. A., Akowe, S. O., Otene, P. M. \& Ikyule, J. M. (2014). An Overview on Intuitionistic Fuzzy Sets, International Journal of Scientific \& Technology Research, 3 (3), $142-145$.

[10] Fono, L. A., Gwet, H. \& Bouchon-Meunier, B. (2007). Fuzzy implication operators for difference operations for fuzzy sets and cardinality-based measures of comparison, European Journal of Operational Research, 183, 314-326.

[11] Fono, L. A., Gwet, H. \& Fotso, S. (2008). On strict lower and upper sections of weakly complete fuzzy pre-orders based on co-implication, Fuzzy Sets and Systems, 159, 22402255 .

[12] Fono, L. A. \& Gwet, H. (2003). On strict lower and upper sections of fuzzy orderings, Fuzzy Sets and Systems, 139, 583-599.

[13] Fono, L. A., Njanpong, G. N., Salles, M. \& Gwet, H. (2009). A binary intuitionistic fuzzy relation: some new results, a general factorization and two properties of strict components, International Journal of Mathematics and Mathematical Sciences, 3-7.

[14] Hinde, C. \& Atanassov, K. T. (2008). On intuitionistic fuzzy negations and intuitionistic fuzzy extended modal operators (Part 2), Proceedings of 4th International IEEE Conference Intelligent Systems, 6-8 Sept. 2008, Varna, Bulgaria, 3, 13-19.

[15] Huawen, L. (1987). Difference operation defined over the intuitionistic fuzzy sets (PhD thesis), School of Mathematics and System Sciences, Shandong University, Jinan, Shandong 250100, China.

[16] Lee, K. H. (2006). First course on fuzzy theory and applications, Springer Science \& Business Media.

[17] Njanpong, G. N. \& Fono, L. A. (2013). Arrow-type results under intuitionistic fuzzy preferences, New Mathematics and Natural Computation, 9 (1), 98-99.

[18] Reiser, R. H. S. \& Bedregal, B. (2013). Interval-valued intuitionistic fuzzy implications construction, properties and representability, Information Sciences, 248, 68-88.

[19] Tripathy, B. K., Jena, S. P. \& Ghosh, S. K. (2013). An intuitionistic fuzzy count and cardinality of intuitionistic fuzzy sets, Malaya Journal of Matematik, 4 (1), 123-133.

[20] Zadeh, L. A. (1965). Fuzzy sets, Information and Control, 8 (3), 338-353. 


\section{Appendix on Fuzzy Operators and Fuzzy Operations}

\section{Fuzzy Sets and Fuzzy Operators}

Definition 11. 1. A fuzzy set $B$ on $X$ is defined by:

$B=\left\{\left(x, \mu_{B}(x)\right) \mid \mu_{B}(x) \in[0,1], \forall x \in X\right\}$ where $\mu_{B}(x)$ is the degree of membership of $x$ in $B$.

2. A fuzzy triangular-norm (fuzzy t-norm) is a binary operation $\top:[0,1] \times[0,1] \longrightarrow[0,1]$ such that for any $x \in[0,1], \top(x, 1)=x$ and $\top$ satisfies commutativity $(\forall a, b \in[0,1], \top(a, b)=$ $\top(b, a))$, monotonicity (increasing) $(\forall a, b, c, d \in[0,1]$, if $a \leq b$ and $c \leq d$, then $\top(a, c) \leq \top(b, d)$ ) and associativity $(\forall a, b, c, \in[0,1], \top(a, \top(b, c)=\top(\top(a, b), c))$.

3. A fuzzy t-conorm is a binary operation $S:[0,1] \times[0,1] \longrightarrow[0,1]$ such that for any $x \in[0,1], S(x, 0)=x$ and $S$ satisfies commutativity, monotonicity (increasing) and associativity.

4. A fuzzy negation $N$ is a non-increasing mapping $N:[0,1] \longrightarrow[0,1]$ with $N(0)=1$ and $N(1)=0$. If $N(N(x))=x, \forall x \in[0,1]$ (i.e. $N$ satisfies the involutive property), then $N$ is called strong fuzzy negation.

5. The dual of a fuzzy $t$-norm $\top$ is a fuzzy $t$-conorm $S$, such that, for all $a, b \in[0,1], \top(a, b)=$ $1-S(1-a, 1-b)$.

6. A fuzzy R-implicator, $I_{\top}$ associated to a t-norm $\top$ is an operator $I_{\top}:[0,1] \times[0,1] \longrightarrow[0,1]$ defined for all $a, b \in[0,1]$ by $I_{\top}(a, b)=\max \{t \in[0,1] \mid \top(a, t) \leq b\}$.

When $\top$ is left continued, we defined the residual implicator $I_{\top}^{1}$, the symetric contraposition implicator $I_{\top}^{2}$, the $Q L$-implicator $I_{\top}^{3}$ and the $S$-implicator $I_{\top}^{4}$ as follows: for all $x, y \in$ $[o, 1], I_{\top}^{1}(x, y)=\max \{t \in[0,1], \top(x, t) \leq y\} ; I_{\top}^{2}(x, y)=1-\min \{t \in[0,1], S(y, t) \geq$ $x\} ; I_{\top}^{3}(x, y)=S(n(x), \top(x, y))$ and $I_{\top}^{4}(x, y)=S(n(x), y)$.

7. A fuzzy co-implicator, $J_{S}$ associated to $S$ is an operator $J_{S}:[0,1] \times[0,1] \longrightarrow[0,1]$ defined for all $a, b \in[0,1]$ by $J_{S}(a, b)=\min \{r \in[0,1] \mid b \leq S(a, r)\}$.

We will require the following useful results to establish the proofs of some basic findings in this research work.

Proposition 9 (See [10-13]). For all $a, b, c \in[0,1]$,

1. $I_{\top}(a, a)=1 ; J_{S}(a, a)=0 ; J_{S}(a, b) \leq b \leq I_{\top}(a, b)$ and $I_{\top}(1, a)=a=J_{S}(0, a)$;

2. $b<a \Longleftrightarrow\left(I_{\top}(a, b)<1\right.$ or $\left.J_{S}(b, a)>0\right)$;

3. $a \leq b \Rightarrow\left\{\begin{array}{l}I_{\top}(b, c) \leq I_{\top}(a, c), \\ I_{\top}(c, a) \leq I_{\top}(c, b) .\end{array} \quad\right.$ and $a \leq b \Rightarrow\left\{\begin{array}{l}J_{S}(b, c) \leq J_{S}(a, c), \\ J_{S}(c, a) \leq J_{S}(c, b) \text {. }\end{array}\right.$

Thus $I_{\top}$ and $J_{S}$ are left decreasing and right increasing operators. 
Proposition 10 (see [13]). Let $S$ and $\top$ be such that, for all $a, b \in[0,1], \top(a, b) \leq 1-S(1-$ $a, 1-b)$. Then

i. for all $a, b \in[0,1], I_{\top}(a, b) \geq 1-J_{S}(1-a, 1-b)$;

ii. if $\top$ and $S$ are dual, then for all $a, b \in[0,1], I_{\top}(a, b)=1-J_{S}(1-a, 1-b)$.

The following examples of fuzzy t-norms and fuzzy t-conorms belonging to a family called Frank t-norms and Frank t-conorms will commonly be referred to in this study (see [10,13]):

Example 7. The Frank t-norms $\left(\top_{F}^{l}\right)_{l \in[0,+\infty]}$ such that, for all $a, b \in[0,1]$,

$$
\top_{F}^{l}(a, b)=\left\{\begin{array}{l}
\top_{M}(a, b)=\min (a, b), \quad \text { if } l=0, \\
\top_{P}(a, b)=a b, \quad \text { if } l=1, \\
\top_{L}(a, b)=\max (a+b-1,0), \quad \text { if } l=+\infty, \\
\log _{l}\left(1+\frac{\left(l^{a}-1\right)\left(l^{b}-1\right)}{l-1}\right), \quad \text { otherwise, }
\end{array}\right.
$$

where $\top_{M}, \top_{P}, \top_{L}$ are the minimum, product and Lukasiewicz fuzzy t-norms, respectively. The Frank t-conorms $\left(S_{F}^{l}\right)_{l \in[0,+\infty]}$ such that, for all $a, b \in[0,1]$,

$$
S_{F}^{l}(a, b)=\left\{\begin{array}{l}
S_{M}(a, b)=\max (a, b), \quad \text { if } l=0, \\
S_{P}(a, b)=a+b-a b, \quad \text { if } l=1, \\
S_{L}(a, b)=\min (a+b, 1), \quad \text { if } l=+\infty, \\
1-\log _{l}\left(1+\frac{\left(l^{1-a}-1\right)\left(l^{1-b}-1\right)}{l-1}\right), \quad \text { otherwise, }
\end{array}\right.
$$

where $S_{M}, S_{P}, S_{L}$ are the maximum, probabilistic sum and Lukasiewicz fuzzy t-conorms, respectively (see $[9,10,13,16,17])$. .

The following are examples of fuzzy R-implications and fuzzy co-implications associated with Frank t-norms and Frank t-conorms respectively.

Example 8. [10, 13, 17]: for all $a, b \in[0,1]$ :

1. Fuzzy R-implication and fuzzy co-implication associated with $\top_{M}$ and $S_{M}$ are respectively given by

$$
I_{\top_{M}}(a, b)= \begin{cases}1, & \text { if } a \leq b, \\ b, & \text { if } a>b\end{cases}
$$

and

$$
J_{S_{M}}(a, b)= \begin{cases}b, & \text { if } a<b, \\ 0, & \text { if } a \geq b .\end{cases}
$$

2. Fuzzy R-implication and fuzzy co-implication associated with $\top_{P}$ and $S_{P}$ are respectively given by

$$
I_{\top_{P}}(a, b)= \begin{cases}1, & \text { if } a \leq b \\ \frac{b}{a}, & \text { if } a>b .\end{cases}
$$


and

$$
J_{S_{P}}(a, b)= \begin{cases}\frac{b-a}{1-a}, & \text { if } a<b, \\ 0, & \text { if } a \geq b .\end{cases}
$$

3. Fuzzy R-implication and fuzzy co-implication associated with $\top_{L}$ and $S_{L}$ are respectively given by

$$
I_{\top_{L}}(a, b)= \begin{cases}1, & \text { if } a \leq b, \\ 1-a+b, & \text { if } a>b .\end{cases}
$$

and

$$
J_{S_{L}}(a, b)=\left\{\begin{array}{l}
b-a, \quad \text { if } a<b \\
0, \quad \text { if } a \geq b
\end{array}\right.
$$

4. Fuzzy R-implication and fuzzy co-implication associated with $\top_{F}^{l}$ and $S_{F}^{l}$ for all $l \in(0,1) \cup$ $(1,+\infty)$ are respectively given by

$$
I_{\top_{F}^{l}}(a, b)=\left\{\begin{array}{l}
1, \quad \text { if } a \leq b, \\
\log _{l}\left(1+\frac{(l-1)\left(l^{b}-1\right)}{l^{a}-1}\right), \quad \text { if } a>b .
\end{array}\right.
$$

and

$$
J_{S_{L}}(a, b)=\left\{\begin{array}{l}
1-\log _{l}\left(1+\frac{(l-1)\left(l^{1-b}-1\right)}{l^{1-a}-1}\right), \quad \text { if } a<b \\
0, \quad \text { if } a \geq b .
\end{array}\right.
$$

\section{Fuzzy Operations of Fuzzy Sets}

Definition 12. Let $A$ and $B$ be any two fuzzy sets defined on $X$. The following operations are defined by associated membership function as follows:

i) Inclusion: $A \subseteq B$ if and only, $\mu_{A}(x) \leq \mu_{B}(x), \forall x \in X$;

ii) Intersection: $A \cap B$ is defined by: $\mu_{A \cap B}(x)=\mu_{A}(x) \wedge \mu_{B}(x), \forall x \in X$;

iii) Union: $A \cup B$ is defined by: $\mu_{A \cup B}(x)=\mu_{A}(x) \vee \mu_{B}(x), \forall x \in X$;

iv) Complement: $A^{c}$ is defined by: $\mu_{A^{c}}(x)=1-\mu_{A}(x), \forall x \in X$.

Where $\vee$ and $\wedge$ are $\max$ and $\min$ respectively. 